\title{
Space Bounds for Adaptive Renaming
}

\section{Maryam Helmi1, Lisa Higham², and Philipp Woelfel \\ University of Calgary \\ Department of Computer Science \\ Calgary, T2N1N4 Alberta, Canada}

\begin{abstract}
We study the space complexity of implementing long-lived and one-shot adaptive renaming from multi-reader multi-writer registers, in an asynchronous distributed system with $n$ processes. As a result of an $f$-adaptive renaming algorithm each participating process gets a distinct name in the range $\{1, \ldots, f(k)\}$ provided $k$ processes participate.

Let $f:\{1, \ldots, n\} \rightarrow \mathbb{N}$ be a non-decreasing function satisfying $f(1) \leq n-1$ and let $d=\max \{x \mid f(x) \leq$ $n-1\}$. We show that any non-deterministic solo-terminating long-lived $f$-adaptive renaming object requires $d+1$ registers. This implies a lower bound of $n-c$ registers for long-lived $(k+c)$-adaptive renaming, which we observe is tight.

We also prove a lower bound of $\left\lfloor\frac{2(n-c)}{c+2}\right\rfloor$ registers for implementing any non-deterministic soloterminating one-shot $(k+c)$-adaptive renaming. We provide two one-shot renaming algorithms: a waitfree algorithm and an obstruction-free algorithm. Each algorithm employs a parameter to depict the tradeoff between space and adaptivity. When these parameters are chosen appropriately, this results in a wait-free one-shot $\left(\frac{3 k^{2}}{2}\right)$-adaptive renaming algorithm from $\lceil\sqrt{n}\rceil+1$ registers, and an obstruction-free one-shot $f$-adaptive renaming algorithm from only $\min \{n, x \mid f(x) \geq 2 n\}+1$ registers.
\end{abstract}

${ }^{1}$ mhelmikh@ucalgary.ca, $+1403210-9416$

${ }^{2}$ higham@ucalgary.ca, +1 403 220-7696

3 woelfel@ucalgary. ca, +1 403 220-7259 


\section{Introduction}

Distributed systems with a large number of processes, such as the Internet, provide services that are typically used by only a small number of processes simultaneously. This is problematic if the time or space used by the service is a function of the size of the name-space of the processes that could use it. The time or space consumed by such applications can be significantly decreased by having each process that wants to use the application first acquire a temporary name from a name space that is adequate to distinguish all the participants, but much smaller than the name-space of the distributed system, and then return the temporary name to the pool when it is finished with the service. This is the role of a shared renaming object. A related application of the renaming object is in operating systems where processes repeatedly acquire and release names that correspond to a limited number of resources [12]. Renaming is an important tool in distributed computing [5] because it enhances the practicality and usefulness of distributed system services. A renaming object may be even more useful if the time and space resources it consumes is a reasonable function of the actual number of processes that are currently either holding, acquiring, or releasing a name. In this paper, we address the renaming problem for the standard asynchronous shared memory model with $n$ processes.

With an $f$-adaptive renaming object, each of the $n$ processes can perform a getName () and return a distinct name in a small domain $\{1, \ldots, f(k)\}$ where $k$ is the number of participants. Herlihy and Shavit [24], and also Rajbaum and Castañeda [13] showed that there is no deterministic, wait-free implementation of $(2 k-2)$-adaptive renaming from multi-reader multi-writer registers. This result also follows from the relationship between the adaptive renaming problem and strong symmetry breaking (SSB): a $(2 k-2)$-renaming algorithm implies a solution to SSB [7], for which there is no deterministic wait-free solution [7, 9, 20]. This impossibility can be circumvented by using randomness or stronger primitives such as compare-and-swaps [2, 4, 16, 28, 29]. The step complexity of deterministic and randomized algorithms has been studied extensively in asynchronous systems (see e.g., [1, 3, 4, 10, 17]). However, there are no previous results on the space complexity of adaptive renaming. Because renaming seems to require that participants discover information about each other, adaptive renaming appears related to $f$-adaptive collect. A collect algorithm is $f$-adaptive to total contention, if its step complexity is $f(k)$, where $k$ is the number of processes that ever took a step. Attiya, Fich and Kaplan [6], proved that $\Omega\left(f^{-1}(n)\right)$ multi-reader multi-writer registers are required for $f$-adaptive collect.

Suppose you have $m$ shared registers available to construct a renaming object for a system with $n$ processes. First we would like to know under what additional conditions such an implementation exists, and when it does, how best to use the $m$ registers. Suppose, when there are $k$ participants, the acquired names are in the range $\{1, \ldots, f(k)\}$. Will $f(k)=k^{c}$ for a small constant $c$ suffice for the application? Must $f(k)$ be closer to $k$, say within a constant? Perhaps it should even be exactly $k$ (tight adaptive renaming)? Does the application need to permit processes to repeatedly acquire and release a name (long-lived renaming), or do processes get a name at most once (one-shot renaming)? How strong a progress guarantee is required? Is the number of participants usually less than some bound $b$ much smaller than $n$ ? If so, is there still some significant likelihood that the number of participants is somewhat bigger than $b$, or is there confidence that the bound $b$ is never, or only very rarely, exceeded? In the rare cases when there are a large number of participants, can the system tolerate name assignments from a very large name space?

In order to study the space complexity implication for these questions, we first generalize the adaptive renaming definition. Both versions (long-lived and one-shot) of b-bounded f-adaptive renaming support the operation get Name (), which returns a name to each invoking process. The long-lived version also supports an operation relName (), which releases the name to the available name domain. Both versions must satisfy 1) no two processes that have completed a getName () and have not started their following relName (), receive the same name, 2) if there are $k \leq b$ processes that have invoked getName () and have not completed their subsequent relName () during an execution of getName () by process $p$, then $p$ returns a name in $\{1, \ldots, f(k)\}$. Observe that for the one-shot case, $k$ is the number of processes that have started a getName () before $p$ completes its getName (). We call the problem of $n$-bounded $f$-adaptive renaming simply $f$-adaptive renaming. The special case when $f(k)=k$ and $b=n$ is called tight renaming. Our goal is to determine the relationships between $b, f(k)$, and $m$ for one-shot versus long-lived, and wait-free versus non-deterministic solo-terminating implementations of adaptive renaming objects from multi-reader/multi-writer registers.

Let $f:\{1, \ldots, n\} \rightarrow \mathbb{N}$ be a non-decreasing function satisfying $f(1) \leq n-1$ and let $d=\max \{x \mid f(x) \leq n-1\}$. Note that if $f(1) \geq n, f$-adaptive renaming is a trivial problem. In this paper we show: 
- At least $d+1$ registers are required to implement any non-deterministic solo-terminating long-lived $d$-bounded $f$-adaptive renaming object.

- At least $\left\lfloor\frac{2(n-c)}{c+2}\right\rfloor$ registers are required to implement any non-deterministic solo-terminating one-shot $(k+c)$ adaptive renaming object where, $c$ is any non-negative integer constant.

- For any $b \leq n$, there is a wait-free one-shot $(b-1)$-bounded $(k(k+1) / 2)$-adaptive renaming algorithm implemented from $b$ bounded registers. When $k \geq b$, the returned names are in the range $\left\{1, \ldots, n+\frac{b(b-1)}{2}\right\}$.

- For any $b \leq n$, there is an obstruction-free one-shot $(b-1)$-bounded $k$-adaptive renaming algorithm implemented from $b+1$ bounded registers. When $k \geq b$, the returned names are in the range $\{1, \ldots, n+b-1\}$.

By using these results and setting $b$ appropriately we then derive the following corollaries:

- A wait-free one-shot $\left(\frac{3 k^{2}}{2}\right)$-adaptive renaming algorithm that uses only $\lceil\sqrt{n}\rceil+1$ registers.

- An obstruction-free one-shot $f$-adaptive renaming algorithm that uses only $\min \{n, x \mid f(x) \geq 2 n\}+1$ registers.

- A tight space lower bound of $n-c$ registers for long-lived $(k+c)$-adaptive renaming for any integer constant $c \geq 0$.

Our lower bound proofs use covering techniques first introduced by Burns and Lynch [11]. The main challenge is to exploit the semantics of the renaming object to force the processes to write to a large number of registers. In the lower bound for the one-shot case, we first build an execution in which some processes are poised to write to (cover) a set of registers. Then we argue that if enough new processes take steps after this, at least one of them must become poised to write to a register not already covered, since, otherwise, the covering processes can obliterate all the traces of the new processes, causing some getName () to return an incorrect result. For the lower bound for the long-lived case, we exploit that fact that processes can perform getName () and relName () repeatedly to build a long execution, where in each inductive step either another register is covered or an available name is used up without being detected by other processes.

\section{Preliminaries}

This section describes our model of computation and the notation, vocabulary and general techniques used in this paper. Previous work by many researchers (for example [8, 11, 18, 19, 23, 27]) has collectively developed similar tools that serve to make our description of results and presentation of proofs precise, concise and clear. Much of the terminology presented in this section is borrowed or adapted from this previous research.

Our computational model is an asynchronous shared memory system consisting of $n$ processes $\mathcal{P}=\left\{p_{1}, \ldots, p_{n}\right\}$ and $m$ shared registers $\mathcal{R}=\left\{R_{1}, \ldots, R_{m}\right\}$. Each process executes code that can access its own independent random number generator and its own private registers as well as the shared registers. Each shared register supports two operations, read and write. Each such operation happens atomically in memory. Processes can only communicate via those operations on shared registers. The algorithm is deterministic if each process' code is deterministic; that is, no process' code contains any random choice.

Informally, an execution arises one step at a time, where a step consists of some process, chosen arbitrarily, executing the next instruction in its code. This instruction could be a shared memory access, or a local memory access, or a local operation including, possibly, a random choice. Notice, however, that after a process takes a shared memory step, the outcome of all its subsequent local operations and random choices up to (but not including) its next shared memory operation is independent of any intervening operations by other processes. Therefore, there is no loss of generality in assuming that a step by a process consists of a single shared memory access (or, initially, its method-call invocation) followed by all its subsequent local operations and random choices, up to the point where that process is poised to execute its next shared memory operation.

A configuration $C$ is a tuple $\left(s_{1}, \ldots, s_{n}, v_{1}, \ldots, v_{m}\right)$, denoting that process $p_{i}, 1 \leq i \leq n$, is in state $s_{i}$ and register $r_{j}$, $1 \leq j \leq m$, has value $v_{j}$. Furthermore the state $s_{i}$ of $p_{i}$ is one in which $p_{i}$ 's next operation is either a shared memory operation or an invocation of a method-call (getName () or relName ()). Configurations will be denoted by capital letters. The initial configuration, where each process' next step is to invoke a method-call, is denoted $C^{*}$.

Given a configuration, $C$, a step from $C$ is a pair of the form $(p, \tau)$ where $p$ is a process identifier, and $\tau$ is a sequence of outcomes that arise from the sequence of all random choices that $p$ makes after completing its pending shared mem- 
ory operation starting from configuration $C$ up to the point where $p$ is poised to do its next shared memory operation. An execution is an alternating sequence of configurations and steps starting and ending with a configuration, and defined inductively as follows. The 0 -step or empty execution starting at $C$ is $(C)$. A $k$-step execution, $k \geq 1$, starting at $C_{0}$ is a sequence $\left(C_{0},\left(q_{1}, \tau_{1}\right), C_{1},\left(q_{2}, \tau_{2}\right), \ldots,\left(q_{k}, \tau_{k}\right), C_{k}\right)$ where

- $\left(C_{0},\left(q_{1}, \tau_{1}\right), C_{1},\left(q_{2}, \tau_{2}\right), \ldots,\left(q_{k-1}, \tau_{k-1}\right), C_{k-1}\right)$ is a $k-1$ step execution starting at $C_{0}$, and

- $\left(q_{k}, \tau_{k}\right)$ is a step from $C_{k-1}$ and $C_{k}$ is the configuration resulting from that step.

An execution is a $k$-step execution for any integer $k \geq 0$. A subsequence, $\sigma=\left(\left(q_{1}, \tau_{1}\right), \ldots,\left(q_{k}, \tau_{k}\right)\right)$, consisting of the steps from an execution starting at $C$ is called a schedule starting at $C$. If $\sigma$ is a schedule starting at $C$, then the execution starting at $C$ arising from $\sigma$ is abbreviated $E=(C ; \sigma)$ and $\sigma(C)$ denotes the final configuration of $E$. If an algorithm is deterministic, then the second component of every step of every execution of the algorithm is empty because there are no random choices. So in this case a schedule is simplified to just a sequence of process identifiers.

A configuration, $C$, is reachable if there exists a finite schedule, $\sigma$, such that $\sigma\left(C^{*}\right)=C$. Let $\sigma$ and $\pi$ be two finite schedules such that $\sigma$ starts at configuration $C$ and $\pi$ starts at $\sigma(C)$. Then $\sigma \pi$ denotes the concatenation of $\sigma$ and $\pi$, and is a schedule starting at $C$. Let $P \subseteq \mathcal{P}$ be a set of processes, and $\sigma$ a schedule. We say $\sigma$ is $P$-only if all the identifiers of processes that appear in $\sigma$ are in $P$. If the set $P$ contains only one process, $p$, then we say $\sigma$ is $p$-only. We denote the set of processes that appear in schedule $\sigma$ by $\operatorname{procs}(\sigma)$.

A deterministic implementation of a method is wait-free if, for any reachable configuration $C$ and any process $p, p$ completes its method call in a finite number of its own steps, regardless of the steps taken by other processes. An implementation of a method is non-deterministic solo-terminating if, for any reachable configuration $C$ and any process $p$, there exists a finite $p$-only schedule, $\sigma$, starting from $C$ such that $p$ has finished its method call in configuration $\sigma(C)[18]$. Non-deterministic solo-termination for deterministic implementations is called obstruction-free.

We say process $p$ covers register $r$ in a configuration $C$, if $p$ writes to $r$ in its next step. A set of processes $P$ covers a set of registers $R$ if for every register $r \in R$ there is a process $p \in P$ such that $p$ covers $r$. If $|P|=|R|$, then we say $P$ exactly covers $R$. Consider a process set $P$ that exactly covers the register set $R$ in configuration $C$. Let $\pi_{P}$ be any permutation which includes exactly one step by each process in $P$. Then the execution $\left(C ; \pi_{P}\right)$ is called a blockwrite by $P$ to $R$. Two configurations $C=\left(s_{1}, \ldots, s_{n}, v_{1}, \ldots, v_{m}\right)$ and $C^{\prime}=\left(s_{1}^{\prime}, \ldots, s_{n}^{\prime}, v_{1}^{\prime}, \ldots, v_{m}^{\prime}\right)$ are indistinguishable to process $p_{i}$ if $s_{i}=s_{i}^{\prime}$ and $v_{j}=v_{j}^{\prime}$ for $1 \leq j \leq n$. Let $P$ be a set of processes, and $\sigma$ any $P$-only schedule starting at configuration $C$. If for every process $p \in P, C$ and $C^{\prime}$ are indistinguishable to $p$, then $\sigma$ is also a schedule starting at $C^{\prime}$ and $\sigma(C)$ and $\sigma\left(C^{\prime}\right)$ are indistinguishable to $p$.

A process $p$ participates in configuration $C$ if in $C, p$ has started a getName () operation and has not completed the following relName (). A process is called idle in configuration $C$ if it does not participate in $C$. A configuration $C$ is called quiescent if, $\forall p \in \mathcal{P}, p$ is idle in $C$. We say process $p$ owns name $x$ in configuration $C$ if in $C, p$ has completed a getName () operation that returned name $x$ and $p$ has not started relName (). Let $C_{0}, \ldots, C_{e}$ be a sequence of configurations arising from execution $E$. The number of participants in $E$ is the maximum over all $i, 0 \leq i \leq e$, of the number of participants in $C_{i}$. Given these definitions, the definition of a renaming object can be stated more precisely as follows. Let $f:\{1, \ldots, n\} \rightarrow \mathbb{N}$ be a non-decreasing function satisfying $f(1) \leq n-1$. Both long-lived and oneshot $b$-bounded $f$-adaptive renaming support the operation getName (). Operation getName () by process $p$ returns a name $x$ to $p$. The long-lived version also supports the operation relName (), which releases the name $x$. Both versions must satisfy 1) there is no reachable configuration in which two processes own the same name, 2) if the number of participants during $p$ 's getName (), $k$, is at most $b$ then, $x \in\{1, \ldots, f(k)\}$. Observe that, properties 1) and 2) imply that $f(k) \geq k$ for all $k \in\{1, \ldots, n\}$.

\section{A Space Lower Bound for Long-Lived Loose Renaming Objects}

For any non-decreasing function $f$ satisfying $f(1) \leq n-1$, let $d$ be the largest integer such that $f(d) \leq n-1$. We prove that at least $d+1$ registers are required for non-deterministic solo-terminating long-lived $f$-adaptive renaming in our system. The proof relies on two lemmas. Lemma 3.1 says that there is no reachable configuration $C$ in which $n-d$ processes own names in the range $\{1, \ldots, n-1\}$ while all of the other $d$ processes are idle and unaware of any of the processes with names. The intuition for this proof is simple: if such a reachable configuration $C$ exists, then there is a configuration reachable from $C$ in which $(n-d)+d=n$ processes all own names in the range $\{1, \ldots, n-1\}$. Lemma 3.2 provides the core of the lower bound argument and the intuition is as follows. Let $C$ be any reachable 
configuration in which fewer than $n-d$ processes own names in the range $\{1, \ldots, n-1\}$ while all of the other $d+1$ processes are idle and unaware of the processes with names. Then there is a reachable configuration from $C$ in which either $d+1$ distinct registers are written, or one more name is owned, and the unnamed processes are again idle and still unaware of the processes with names. Since the initial configuration has no processes with names, and all processes are idle, we can apply Lemma 3.2 repeatedly until either we have exactly covered $d+1$ registers or we reach a configuration in which $n-d-1$ processes own names in the range $\{1, \ldots, n-1\}$. Since, according to Lemma 3.1 , we cannot get beyond an $(n-d-1)$-invisibly-named configuration, we must eventually exactly cover $d+1$ registers, completing the proof. We will see, in the formal proof, that the result applies even when the renaming implementation is $(d+1)$-bounded.

The definitions and lemmas that follow refer to any non-deterministic solo-terminating implementation from shared registers of a long-lived $f$-adaptive renaming object. For a configuration $C$ and a set of processes $Q$, we say $Q$ is invisible in $C$, if there is a reachable quiescent configuration $D$ such that $C$ and $D$ are indistinguishable to all processes in $\bar{Q}$. If the set $Q$ contains only one process $q$, then we say process $q$ is invisible. Configuration $C$ is called $\ell$-invisiblynamed, if there is a set $Q$ of $\ell$ processes, such that in $C$ every process in $Q$ owns a name in $\{1, \ldots, n-1\}$ and $Q$ is invisible.

Lemma 3.1. For the largest integer $d$ satisfying $f(d) \leq n-1$, there is no reachable ( $n$-)-invisibly-named configuration.

Proof. By way of contradiction, suppose that there exists a set $Q$ of $n-d$ processes such that in configuration $C$, all processes in $Q$ are invisible and own names in the range $\{1, \ldots, n-1\}$. Since $Q$ is invisible in $C$, there is a reachable quiescent configuration $D$ such that $D$ and $C$ are indistinguishable to $\bar{Q}$. Let $\sigma$ be a $\bar{Q}$-only schedule such that in execution $(D ; \sigma)$, all processes in $\bar{Q}$ perform a complete get Name (). Because $|\bar{Q}|=d$ all processes in $\bar{Q}$ get names in the range $\{1, \ldots, f(d)\} \subseteq\{1, \ldots, n-1\}$. Since $C$ and $D$ are indistinguishable to $\bar{Q}$, all processes in $\bar{Q}$ perform a complete getName () during $(C ; \sigma)$ and get names in the range $\{1, \ldots, f()\}$ as well. Therefore in configuration $\sigma(C)$ all processes in $Q \cup \bar{Q}$ have names in the range $\{1, \ldots, n-1\}$. However $|Q \cup \bar{Q}|=n$. This is a contradiction because this implies that the acquired names are not distinct.

The intuition for Lemma 3.2 is as follows. Recall that in an $\ell$-invisibly-named configuration, $\ell$ processes have names, the $n-\ell$ others are idle and unaware of the presence of the invisibly-named processes, and no register is covered. Starting from this configuration we select one process at a time from the set of idle processes and let it execute until either it covers a register not already covered, or it gets a name without covering a new register. We continue this construction as long as the selected process covers a new register. If we reach $d+1$ processes covering distinct registers we are done. Otherwise, we reached a configuration in which one more process holds a name. Furthermore, we can obliterate the trace of this process with the appropriate block write, and then let all other non-idle processes complete their getName () methods and the following relName (). This takes us to an $(\ell+1)$ invisibly-named configuration.

Lemma 3.2. Let $d$ be the largest integer such that $f(d) \leq n-1$. For any $0 \leq \ell \leq n-d-1$ and any reachable $\ell$-invisibly-named configuration $C$, there exists a schedule $\sigma$, where $|\operatorname{procs}(\sigma)| \leq d+1$, and either

- in configuration $\sigma(C)$ at least $d+1$ distinct registers are exactly covered; or

- configuration $\sigma(C)$ is $(\ell+1)$-invisibly-named.

Proof. Let $C$ be an $\ell$-invisibly-named configuration, and let $Q$ be the set of $\ell$ processes that are invisible in $C$. Let $D$ be a quiescent configuration that is indistinguishable from $C$ for all processes in $\bar{Q}$. First, we inductively construct a sequence of schedules $\delta_{0}, \delta_{1}, \ldots$ until we have constructed $\delta_{\text {last }}$ such that in $\delta_{\text {last }}(C)$ either

a) $d+1$ registers are exactly covered, or,

b) $(\ell+1)$ processes own names in $\{1, \ldots, n-1\}$.

We maintain the invariant that for each $i \in\{0, \ldots$, last $\}$ in configuration $\delta_{i}(C)$, a set $P_{i}$ of $i$ processes exactly covers a set $L_{i}$ of $i$ distinct registers, $P_{i} \cap Q=\emptyset$, and $\delta_{i}$ is $P_{i}$-only. Let $\delta_{0}$ be the empty schedule. Then in configuration $\delta_{0}(C)=C$, no register is covered, so the invariant is true for $P_{0}=L_{0}=\emptyset$.

Now consider $i \geq 0$. If $a$ ) or $b$ ) holds for $\delta_{i}$, we let last $=i$ and are done. Otherwise, since in $\delta_{i}(C)$ a set $L_{i}$ of $i$ distinct registers is covered, we have $i \leq d$. We construct $\delta_{i+1}$ as follows. Select $p \in \overline{P_{i} \cup Q}$. Let $\gamma$ be the shortest $p$-only schedule such that either 
1) $p$ does a complete getName () in execution $\left(\delta_{i}(C) ; \gamma\right)$, or

2) in configuration $\gamma\left(\delta_{i}(C)\right)$, $p$ covers a register $r \notin L_{i}$.

Let $\delta_{i+1}$ be $\delta_{i} \gamma$. First assume case 1) happens. By construction the process that performs $\delta_{1}$ does not write to any register. If $i=0$ and $p$ does a complete getName () in execution $\left(\delta_{0}(C) ; \gamma\right)$, then last $=1$ and we are done. For any $i \geq 1$, because $Q$ is invisible to $p$, in $\left(\delta_{i}(C) ; \gamma\right) p$ becomes aware of at most the $i-1 \leq d$ other processes in $P_{i}$. Since $f(d) \leq n-1, p$ gets a name in $\{1, \ldots, n-1\}$, and thus in configuration $\delta_{i+1}(C)$ all processes in $Q \cup\{p\}$ own names in $\{1, \ldots, n-1\}$ and $|Q \cup\{p\}|=\ell+1$. So condition $b)$ is achieved, the construction stops and $\delta_{\text {last }}=\delta_{i+1}$.

Now suppose case 2) happens. If $i+1=d+1$, then condition $a$ ) is achieved, the construction stops and $\delta_{\text {last }}=\delta_{i+1}$. Otherwise, the invariant remains satisfied for $L_{i+1}=L_{i} \cup\{r\}$ and $P_{i+1}=P_{i} \cup\{p\}$. Clearly, after at most $d+1$ steps either $a$ ) or $b$ ) is achieved.

Now, using schedule $\delta_{\text {last }}$ we construct schedule $\sigma$. If $\delta_{\text {last }}(C)$ satisfies $a$ ), let $\sigma=\delta_{\text {last }}$ and the lemma holds.

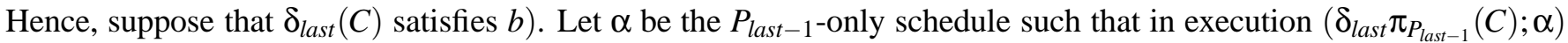
every process $q \in P_{\text {last }-1}$ completes its pending getName () operation and performs a complete relName (). During execution $\left(C ; \delta_{\text {last }}\right)$ only registers in $L_{\text {last }-1}$ were written and in configuration $\delta_{\text {last }}(C), P_{\text {last }-1}$ exactly covers these registers. Because $\delta_{\text {last }}=\delta_{\text {last-1 }} \gamma$ for some $p$-only postfix $\gamma$ of $\delta_{\text {last }}$, after a block write by $P_{\text {last }-1}$, configurations $\delta_{\text {last }} \pi_{P_{\text {last }-1}}(C)$ and $\delta_{\text {last }-1} \pi_{P_{\text {last }-1}}(C)$ are indistinguishable to $\overline{Q \cup\{p\}}$. Since $C$ and $D$ are indistinguishable to $\bar{Q}$, configurations $\delta_{\text {last }-1} \pi_{P_{\text {last }-1}}(C)$ and $\delta_{\text {last }-1} \pi_{P_{\text {last }-1}}(D)$ are also indistinguishable to $\bar{Q}$. So, configurations $\delta_{\text {last }} \pi_{P_{\text {last }-1}}(C)$ and $\delta_{\text {last }-1} \pi_{P_{\text {last }-1}}(D)$ are indistinguishable to $\overline{Q \cup\{p\}}$. Hence, configurations $\delta_{\text {last }} \pi_{P_{\text {last }-1}} \alpha(C)$ and $\delta_{\text {last }-1} \pi_{P_{\text {last }-1}} \alpha(D)$ are indistinguishable to $\overline{(Q \cup\{p\})}$. Since $\delta_{\text {last }-1} \pi_{P_{\text {last }-1}} \alpha(D)$ is quiescent, configuration $\delta_{\text {last }} \pi_{P_{\text {last }-1}} \alpha(C)$ is an $(\ell+1)$ invisibly-named configuration. Therefore, the lemma holds for $\sigma=\delta_{\text {last }} \pi_{P_{\text {last }-1}} \alpha$.

Theorem 3.3. Let $d$ be the largest integer such that $f(d) \leq n-1$. Any non-deterministic solo-terminating implementation of a long-lived $d$-bounded $f$-adaptive renaming object requires at least $d+1$ registers.

Proof. Note that $C^{*}$ is a reachable 0 -invisibly-named configuration. We iteratively construct a sequence of schedules $\sigma_{0}, \sigma_{1}, \ldots, \sigma_{\text {last }}$ as follows: If $0 \leq i \leq n-d$ and $C_{i}$ is a reachable $i$-invisibly-named configuration, we apply Lemma3.2 to obtain a schedule $\sigma_{i},\left|\operatorname{procs}\left(\sigma_{i}\right)\right| \leq d+1$, such that $C_{i+1}=\sigma_{i}\left(C_{i}\right)$ is either an $(i+1)$-invisibly-named configuration, or in $C_{i+1}$ at least $d+1$ distinct registers are covered. In the latter case we let last $=i+1$ and finish the iterative construction. By Lemma 3.1, there is no $(n-d)$-invisibly-named configuration. Hence if the iterative construction reaches a $(n-d-1)$-invisibly-named configuration, by Lemma 3.2, there is a reachable configuration, in which $d+1$ registers are covered.

Corollary 3.1. Let $c \in\{1, \ldots, n-1\}$ and $b=n-c$. Any non-deterministic solo-terminating implementation of a long-lived b-bounded $(k+c)$-adaptive renaming object requires at least $b$ registers.

\section{A Space Lower Bound for One-shot Additive Loose Renaming}

In one-shot renaming, each process is constrained to call getName () at most once (and does not invoke relName ()), which imposes a severe restriction on the techniques available for proving lower bounds. In particular, constructions that rely on processes repeatedly getting and releasing names cannot be used for one-shot lower bounds. We observed however, that a straightforward linear lower bound for tight renaming actually applies even for one-shot adaptive renaming. Thus, we are motivated to study one-shot renaming objects with looseness constrained by a constant, specifically $k$-renaming and $(k+c)$-renaming. We refer to one-shot $(k+c)$-renaming object as an additive loose renaming object, where $k$ is the number of participants and $c \geq 0$ is an integer constant. For the case $c=0$, it is called an adaptive tight renaming object.

Our lower bound proof has a recursive structure and it relies on a generalization of additive loose renaming as follows. For any set $T \subset\{1, \ldots, k+c\}$ where $|T| \leq c$, a $[(k+c) \backslash T]$-renaming object for $k$ processes requires that each participating process returns a unique name from the range $\{1, \ldots, k+c\} \backslash T$.

Lemma 4.1. Any implementation of $[(k+c) \backslash T]$-renaming uses at least as many registers as an implementation of $[(k+c-|T|) \backslash \emptyset]$-renaming. 
Proof. Let $A$ be a $[(k+c) \backslash T]$-renaming algorithm. Then we construct $[(k+c-|T|) \backslash \emptyset]$-renaming algorithm $A^{\prime}$ from $A$ without any additional registers as follows. If $A$ returns name $x$, then $A^{\prime}$ returns $x-|\{t \in T \mid t \leq x\}|$. Since $A$ returns distinct names in the range $\{1, \ldots, k+c\} \backslash T$, obviously $A^{\prime}$ returns distinct names in the range $\{1, \ldots, k+c-|T|\}$.

A process is called startable in configuration $C$ if in $C$, it has not started a getName (). Since in one-shot renaming, there is no relName () operation, in our proofs in this section instead of using quiescent configurations we are interested in configurations in which each process either has completed its getName () operation or it has not started a get Name (). We call such configurations, quiet configurations.

Lemma 4.2. Let $D$ be a reachable quiet configuration with $n^{\prime} \geq c+2$ startable processes. For every startable process $p$, let $\sigma_{p}$ denote a p-only schedule such that p performs a complete getName () in execution $\left(D ; \sigma_{p}\right)$. Let $Q$ be any subset of startable processes of size $c+1$, then there exists a process $q \in Q$ such that $q$ writes to a register during $\left(D ; \sigma_{q}\right)$.

Proof. Let $X$ be the set of processes that own names in configuration $D$. Then processes in $X$ own names in range $\{1, \ldots,|X|+c\}$. Let $Q=\left\{q_{1}, \ldots, q_{c+1}\right\}$. By way of contradiction assume that there is no process $q \in Q$ such that $q$ writes to a register during $\left(D ; \sigma_{q}\right)$. Then for all $i, 1 \leq i \leq c+1$, configurations $\sigma_{q_{1}} \ldots \sigma_{q_{c+1}}(D)$ and $\sigma_{q_{i}}(D)$ are indistinguishable to $q_{i}$. Let $q^{\prime}$ be a startable process not in $Q$. Hence, $\sigma_{q_{1}} \ldots \sigma_{q_{c+1}} \sigma_{q^{\prime}}(D)$ and $\sigma_{q^{\prime}}(D)$ are indistinguishable to $q^{\prime}$. Therefore, all processes in $Q$ plus $q^{\prime}$ return names from $\{1, \ldots,|X|+1+c\}$ in execution $\left(D ; \sigma_{q_{1}} \ldots \sigma_{q_{c+1}} \sigma_{q^{\prime}}\right)$. This is a contradiction because $|X|+c+2$ processes receive names from a set of size $|X|+c+1$ implying that they cannot be assigned distinct names.

Lemma 4.3. Let $D$ be a reachable configuration in which:

- a set $Q$ of at least $c+1$ processes covers a set of $\ell \geq 1$ registers,

- there exists a set $Q^{\prime} \subseteq Q$ of size c +1 such that no process in $Q^{\prime}$ has written to a register and,

- there is a set of $c+1$ startable processes $P$, disjoint from $Q$.

Then, there is a P-only schedule $\sigma_{P}$ such that at least $\ell+1$ registers are covered in $\sigma_{P}(D)$.

Proof. Let $X$ be the set of processes that own names in configuration $D$. Since no process in $Q^{\prime}$ has written to a register, processes in $X$ own names in range $\left\{1, \ldots,|X|+|Q|-\left|Q^{\prime}\right|+c\right\}$. Let $L$ be the set of registers covered by $Q$ and $Q^{\prime \prime} \subseteq Q$ be a set of processes that exactly covers $L$. Let $\widehat{\sigma_{P}}$ be a $P$-only schedule such that in execution $\left(D ; \widehat{\sigma_{P}}\right)$ all processes in $P$ complete their getName () operations. Then all processes in $P$ return names from $\left\{1, \ldots,|X|+|Q|-\left|Q^{\prime}\right|+|P|+c\right\}=$ $\{1, \ldots,|X|+|Q|+c\}$ in execution $\left(D ; \widehat{\sigma_{P}}\right)$. Suppose that in execution $\left(D ; \widehat{\sigma_{P}}\right)$, there is a process in $P$ that writes to a register not in $L$. Then let $\sigma_{P}$ be the shortest prefix of $\widehat{\sigma_{P}}$ such that a register $r \notin L$ is covered by a process in $P$. Hence in configuration $\sigma_{P}(D), L$ is covered by $Q$ and $r$ is covered by $P$. Thus we are done. Therefore, assume that in execution $\left(D ; \widehat{\sigma_{P}}\right)$ all processes in $P$ write only to $L$. Let $\pi_{Q^{\prime \prime}}$ be a block-write to $L$ by $Q^{\prime \prime}$. Let $\sigma_{Q}$ be a $Q-$ only schedule such that in execution $\left(\sigma_{P} \pi_{Q^{\prime \prime}}(D) ; \sigma_{Q}\right)$ all processes in $Q$ complete their get Name () operations. Since configurations $\sigma_{P} \pi_{Q^{\prime \prime}}(D)$ and $\pi_{Q^{\prime \prime}}(D)$ are indistinguishable to all processes in $Q$, processes in $Q$ return names from $\{1, \ldots,|X|+|Q|+c\}$ in execution $\left(\sigma_{P} \pi_{Q^{\prime \prime}}(D) ; \sigma_{Q}\right)$. This is a contradiction because $|X|+|Q|+|P|=|X|+|Q|+c+1$ processes receive names from a set of size $|X|+|Q|+c$ implying that they cannot be assigned distinct names.

Lemma 4.4. Let $D$ be a reachable configuration in which:

- a set $P$ of processes exactly covers a set of $\ell \geq 1$ registers,

- there exists a process $q \in P$ such that $q$ has not written to any register and,

- there exists a set of $n^{\prime} \geq c$ startable processes.

Then, there exists a configuration reachable from $D$, in which at least $\ell+\left\lfloor\frac{n^{\prime}-c}{c+1}\right\rfloor$ registers are covered. 
Proof. Let $\mathcal{P}^{\prime}$ be the set of all startable processes in $D$ and $Q \subseteq \mathcal{P}^{\prime}$ be a set of $c$ processes. Then in configuration $D$, processes in $Q \cup P$ cover a set of $\ell$ registers where $|Q \cup P| \geq c+1$ and, no process in set $Q \cup\{q\}$ has written to a register. Hence using startable processes in $\mathcal{P}^{\prime} \backslash Q$, we can inductively apply Lemma 4.3 , until we reach a configuration in which $\ell+\left\lfloor\frac{n^{\prime}-c}{c+1}\right\rfloor$ registers are covered.

Lemma 4.5. Let $A$ be an non-deterministic solo-terminating implementation of one-shot adaptive tight renaming. Let $D$ be any reachable quiet configuration with $n^{\prime} \geq 2$ startable processes. Then there is an execution of $A$, starting from $D$ that requires at least $n^{\prime}$ registers.

Proof. Let $p$ be a startable process and $\widehat{\sigma_{p}}$ be a $p$-only schedule such that in $\left(D ; \widehat{\sigma_{p}}\right), p$ completes its getName (). Then by Lemma 4.2 $p$ writes to a register. Let $\sigma_{p}$ be the shortest prefix of $\widehat{\sigma_{p}}$ such that in $\left(D ; \sigma_{p}\right), p$ covers a register. Then by Lemma 4.4 there exists a configuration reachable from $\sigma_{p}(D)$, in which at least $1+n^{\prime}-1=n^{\prime}$ registers are covered.

In Lemma 4.6, we show at least $\left\lfloor\frac{2\left(n^{\prime}-c\right)}{c+2}\right\rfloor$ registers are required for a non-deterministic solo-terminating implementation of one-shot $(k+c)$-adaptive renaming starting from a quiet configuration with $n^{\prime} \geq 2 c+2$ startable processes. The intuition for this lemma is as follows. We prove the lemma by induction on $c$. Starting from any quiet configuration, first we select a set $Q$ of $c+1$ startable processes such that one of them writes to a register in a solo-run and we stop it immediately before it writes. Then we choose a process $p$ not in $Q$ and run it until it covers a new register. If we succeed, we select another startable process not in $Q$. We might not succeed for two reasons. First, we are out of startable processes in which case we are done. Second, process $p$ completes its getName () and only writes to the set of covered registers. Then Lemma 4.4 provides a lower bound. Furthermore, starting from this configuration, if the set of covering processes perform a block-write and cover $p$ 's trace, then no other process distinguishes this execution from one in which $p$ has not run at all. Therefore by Lemma 4.1, our problem reduces to one-shot $(k+c-1)$-adaptive renaming. Hence we can invoke the induction hypothesis and conclude a second lower bound. Our final lower bound is the maximum of these two lower bounds.

Lemma 4.6. Let $A$ be a non-deterministic solo-terminating implementation of one-shot $(k+c)$-adaptive renaming. Let $D$ be any reachable quiet configuration with $n^{\prime} \geq 2 c+2$ startable processes. Then there is an execution of $A$, starting from $D$ that requires at least $\left\lfloor\frac{2\left(n^{\prime}-c\right)}{c+2}\right\rfloor$ registers.

Proof. Let $\mathcal{P}^{\prime}$ be the set of startable processes in $D$. We prove the lemma by induction on $c$. For the base case $c=0$, by Lemma 4.5, the hypothesis is true. Suppose that the induction hypothesis is true for $c-1 \geq 0$. Since, $\left|\mathcal{P}^{\prime}\right|>c+1$, by Lemma 4.2 there is a process $q \in \mathcal{P}^{\prime}$ that writes to a register in a solo-execution starting from $D$. Let $\sigma_{q}$ be the shortest $q$-only schedule such that in configuration $\sigma_{q}(D)$, there is a register $r$ covered by $q$. Let $Q \subseteq\left(\mathcal{P}^{\prime} \backslash\{q\}\right)$ be a set of $c$ processes.

First, we inductively construct a sequence of schedules $\delta_{1}, \delta_{2}, \ldots$ until we have constructed $\delta_{\ell}$ such that in $\delta_{\ell}(D)$ either

a) $\left|\mathcal{P}^{\prime} \backslash Q\right|$ registers are covered or,

b) a process $q^{\prime}$ in $\mathcal{P}^{\prime} \backslash Q$ has completed its getName () and has written only to registers covered by other processes.

We maintain the invariant that for each $i \in\{1, \ldots, \ell\}$ in configuration $\delta_{i}(D)$, a set $P_{i} \subseteq\left(\mathcal{P}^{\prime} \backslash Q\right)$ of $i$ processes covers a set $L_{i}$ of $i$ distinct registers and $\delta_{i}$ is $P_{i}$-only.

Let $\delta_{1}$ be $\sigma_{q}$. Then in configuration $\delta_{1}(D)$, one register is covered, so the invariant is true for $P_{1}=\{q\}$ and $L_{1}=\{r\}$.

Now consider $i \geq 1$. If $a$ ) or $b$ ) holds for $\delta_{i}$, we let $\ell=i$ and the construction stops. Furtheremore in case $b$ ), let $q^{\prime}$ be the process that completes its get Name ().

Otherwise, since in $\delta_{i}(D)$ a set $L_{i}$ of $i$ distinct registers is covered, we construct $\delta_{i+1}$ as follows. Select $p \in$ $\mathcal{P}^{\prime} \backslash\left(P_{i} \cup Q\right)$. Let $\gamma$ be the shortest $p$-only schedule such that either

1) $p$ does a complete getName () in execution $\left(\delta_{i}(D) ; \gamma\right)$ and only writes to $L_{i}$, or

2) in configuration $\gamma\left(\delta_{i}(D)\right)$, $p$ covers a register $r^{\prime} \notin L_{i}$.

Let $\delta_{i+1}$ be $\delta_{i} \gamma$. First assume case 1) happens. Then condition $b$ ) is achieved, the construction stops and we let $\delta_{\ell}=\delta_{i+1}$ and $q^{\prime}=p$. Now suppose case 2) happens. If $i+1=\left|\mathcal{P}^{\prime} \backslash Q\right|$, then condition $a$ ) is achieved, the construction stops and $\delta_{\ell}=\delta_{i+1}$. Otherwise, the invariant remains satisfied for $L_{i+1}=L_{i} \cup\left\{r^{\prime}\right\}$ and $P_{i+1}=P_{i} \cup\{p\}$. Clearly, after at most $\left|\mathcal{P}^{\prime} \backslash Q\right|$ steps either $a$ ) or $b$ ) is achieved. 
In case $a$ ), in configuration $\delta_{\ell}(D), \ell=n^{\prime}-c$ registers are covered so the lemma holds in this case. Now suppose case $b$ ) happens. In configuration $\delta_{\ell-1}(D)$, a set of $\ell-1$ registers (i.e $L_{\ell-1}$ ) are covered exactly by a set of processes $P_{\ell-1}$ and process $q$ in $P_{\ell-1}$ has not written to any registers. Furthermore in configuration $\delta_{\ell-1}(D)$, all processes in $P^{\prime} \backslash P_{\ell-1} \supseteq Q$ are startable. Therefore, by Lemma 4.4 there is a configuration reachable from $\delta_{\ell-1}(D)$ in which at least $\ell-1+\left\lfloor\frac{n^{\prime}-\ell+1-c}{c+1}\right\rfloor$ registers are covered. Let $\pi_{P_{\ell-1}}$ be a block-write by $P_{\ell-1}$. Let $\sigma_{P_{\ell-1}}$ be a $P_{\ell-1}$-only schedule such that in execution $\left(\delta_{\ell} \pi_{P_{\ell-1}}(D) ; \sigma_{P_{\ell-1}}\right)$ all processes in $P_{\ell-1}$ complete their getName (). Let $x$ be the name taken by $q^{\prime}$ in execution $\left(D ; \delta_{\ell}\right)$. Let $X$ be the set of processes that own names in configuration $D$. Since in configuration $\delta_{\ell}(D)$, only processes in $X \cup P_{\ell}$ have invoked a getName ( ), $x \in\{1, \ldots,|X|+\ell+c\}$. Note that $\delta_{\ell} \pi_{P_{\ell-1}} \sigma_{P_{\ell-1}}(D)$ and $\delta_{\ell-1} \pi_{P_{\ell-1}} \sigma_{P_{\ell-1}}(D)$ are indistinguishable to all processes except $q^{\prime}$. Hence in any $\left(\mathcal{P}^{\prime} \backslash P_{\ell}\right)$-only execution starting from $\delta_{\ell-1} \pi_{P_{\ell-1}} \sigma_{P_{\ell-1}}(D)$, names returned by processes in $\mathcal{P}^{\prime} \backslash P_{\ell}$ are in $\{1, \ldots, k+c\} \backslash\{x\}$ where $k \geq|X|+\ell$ and therefore $\{x\} \subset\{1, \ldots, k+c\}$. Thus by Lemma 4.1, starting at $\delta_{\ell-1} \pi_{P_{\ell-1}} \sigma_{P_{\ell-1}}(D)$, algorithm $A$ requires as many registers as a $(k+c-1)$-renaming algorithm starting at a quiet configuration with $\left|\mathcal{P}^{\prime}-P_{\ell}\right|=n^{\prime}-\ell$ startable processes. Therefore, by the induction hypothesis there is a reachable configuration from $\delta_{\ell-1} \pi_{P_{\ell-1}} \sigma_{P_{\ell-1}}(D)$, in which at least $\left\lfloor\frac{2\left(n^{\prime}-\ell-c+1\right)}{c+1}\right\rfloor$ registers are covered.

The minimum of $\ell-1+\left\lfloor\frac{n^{\prime}-\ell+1-c}{c+1}\right\rfloor$ and $\left\lfloor\frac{2\left(n^{\prime}-\ell-c+1\right)}{c+1}\right\rfloor$, is maximized when $\ell-1+\left\lfloor\frac{n^{\prime}-\ell+1-c}{c+1}\right\rfloor=\left\lfloor\frac{2\left(n^{\prime}-\ell-c+1\right)}{c+1}\right\rfloor$. Hence, $\ell=\left\lfloor\frac{n^{\prime}-c}{c+2}\right\rfloor-1$. Therefore the algorithm requires at least $\left\lfloor\frac{2\left(n^{\prime}-c\right)}{c+2}\right\rfloor$ registers.

Theorem 4.7. Any non-deterministic solo-terminating implementation of one-shot $(k+c)$-adaptive renaming requires at least $\left\lfloor\frac{2(n-c)}{c+2}\right\rfloor$ registers.

Proof. The initial configuration is a quiet configuration with $n$ startable processes. Hence, by Lemma 4.6, there is an execution, starting from the initial configuration that requires at least $\left\lfloor\frac{2(n-c)}{c+2}\right\rfloor$ registers.

Observe that by setting $c=0$, it follows from Theorem 4.7 that any non-deterministic solo-terminating implementation of one-shot adaptive tight renaming requires $n$ registers. Since the number of startable processes is initially $n$, next corollary also follows from Lemma 4.6

Corollary 4.1. Any non-deterministic solo-terminating implementation of one-shot adaptive tight renaming requires at least $n$ registers.

\section{Wait-Free One-shot $(b-1)$-Bounded $(k(k+1) / 2)$-Adaptive Renaming}

In this section we present a wait-free one-shot $(b-1)$-bounded $(k(k+1) / 2)$-adaptive renaming algorithm from $b$ registers. Since 0-bounded adaptive renaming is a trivial problem, we assume that $b \geq 2$.

The algorithms in this section employ a set $\mathcal{R}=\{R[0], \ldots, R[b-1]\}$ of shared atomic registers. In our proofs, a register configuration is a tuple $\left(V_{0}, \ldots, V_{b-1}\right)$, denoting that register $R[i], 0 \leq i \leq b-1$, has value $V_{i}$. The proofs focus on just the sub-sequence of register configurations produced by an execution. Specifically, given an execution $E=\left(C_{0} ; \sigma\right)$, let write schedule $\widehat{\sigma}$ be the sub-sequence of $\sigma$ that produces write steps in $\left(C_{0} ; \sigma\right)$. Execution $E$ gives rise to the sequence of register configurations $\Gamma_{E}=C_{0}, C_{1}, \ldots$ such that the $i$-th step of $\hat{\sigma}$ is a write that changes register configuration $C_{i-1}$ to register configuration $C_{i}$. For any scan operation $s$ in $E$, define index $(s)=i$, if $s$ occurs in $E$ between $C_{i}$ and $C_{i+1}$ in $\Gamma_{E}$. For any write operation $w$ in $E$, define $\operatorname{index}(w)=i$, if $w$ is the $i$-th step of $\widehat{\sigma}$. Notice that the view returned by a scan with index $i$ is equal to $C_{i}$. A register configuration $C=\left(V_{0}, \ldots, V_{b-1}\right)$ is consistent if $V_{0}=\cdots=V_{b-1}$ in which case we say $V_{0}$ is the content of $C$. Let $C_{i}$ and $C_{j}$ be register configurations in the sequence $\Gamma_{E}=C_{0}, C_{1}, \ldots$ such that $i \leq j$. Interval $[i, j]$ denotes the sub-sequence of steps in execution $E$ that begins at write operation $w$ where index $(w)=i$, and ends immediately after write operation $u$ where index $(u)=j$. We use $v_{C}(R)$ to denote the content of register $R$ in configuration $C$. A local variable $x$ in these algorithms is denoted by $x_{p}$ when it is used in the method call invoked by process $p$.

\section{$5.1(b-1)$-Bounded $(k(k+1) / 2)$-Adaptive Renaming Using Atomic Scan}

Fig. 1 presents a wait-free implementation of a one-shot $(b-1)$-bounded $(k(k+1) / 2)$-adaptive renaming algorithm assuming an atomic scan operation. In Section 5.2, we show how to remove this assumption. 
shared: $\mathcal{R}=R[0, \ldots, b-1]$ is an array of multi-writer multi-reader registers, each register is initialized to $\emptyset$ local: An array $r[0, \ldots, b-1] ;$ pos $\in\{0, \ldots, b-1\}$ initialized to $0 ; S$ initialized to $\{i d\}$;

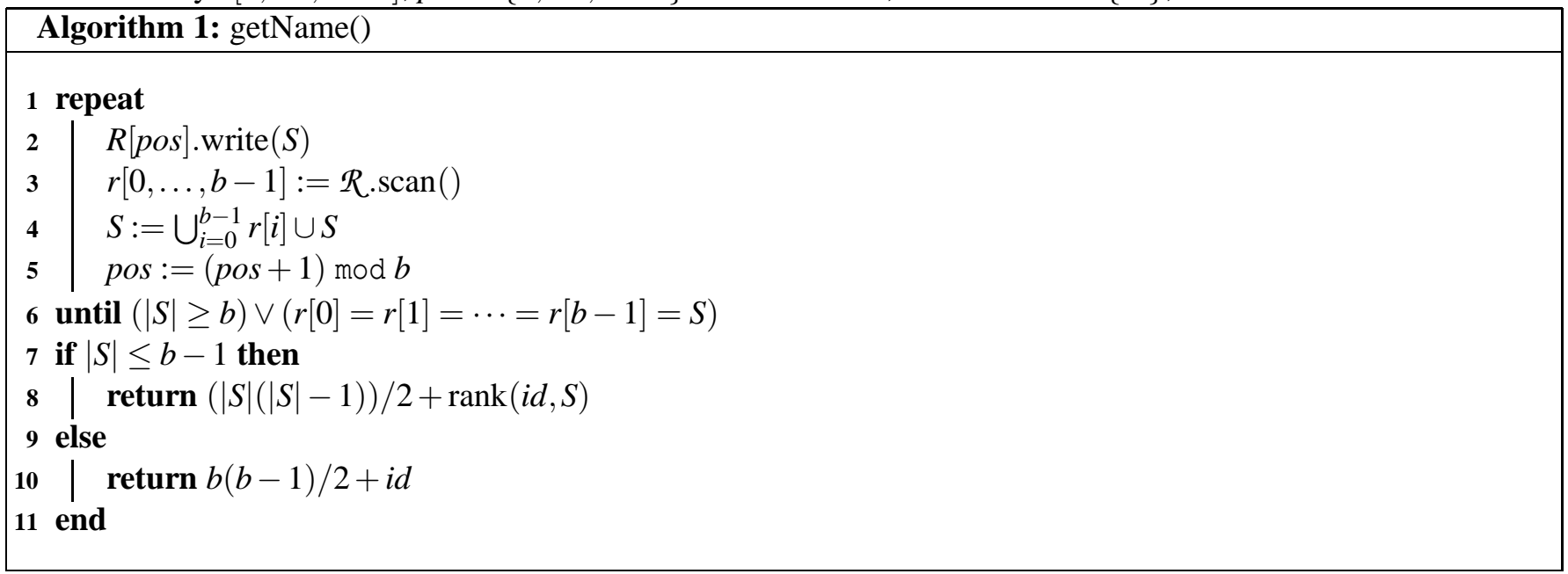

Figure 1: $(b-1)$-Bounded $(k(k+1) / 2)$-Adaptive Renaming Using Atomic Scan

Each process maintains a set of processes, $S$, that it knows are participating including itself, and alternately executes write and scan operations. In the write operation, it writes $S$ to the next register after where it last wrote, in cyclic order through the $b$ registers. After each of its scan operations, it updates $S$ to all the processes it sees in the scan together with the processes already in its set. The process stops with an assigned name when either its scan shows exactly its own set, $S$, in every register, or $S$ has grown to size at least $b$. If $|S|$ is less than $b$, its name is based on $|S|$ and its rank in $S$, where $\operatorname{rank}(i d, S)=|\{i \mid(i \in S) \wedge(i \leq i d)\}|$. If $|S|$ is $b$ or greater, it returns a safe but large name.

\section{Correctness and space complexity.}

Since it is clear that the algorithm in Fig. 1 uses $b$ registers, the space complexity will follow immediately after confirming that it is a correct adaptive renaming algorithm. The correctness of this algorithm relies on the fact that if any two processes return names based on a set of size $s<b$, then they have the same set. The main component of the proof is to establish this fact.

Observation 5.1. For any write operation with value $S$ by process $p, p \in S$.

Lemma 5.2. For any execution $E$, let $C_{a}$ be a consistent register configuration with content $\widehat{S}$. For any register configuration $D$ following $C_{a}$ in $E$, define $\mathcal{T}_{D}=\left\{R \in \mathcal{R} \mid \widehat{S} \nsubseteq v_{D}(R)\right\}$. Then there exists a one-to-one function $f_{D}$ : $\mathcal{T}_{D} \rightarrow \mathcal{P}$ satisfying, $\forall R \in \mathcal{T}_{D}, f_{D}(R) \in \mathcal{v}_{D}(R)$ and $f_{D}(R)$ performs at least one write in the execution interval between $C_{a}$ and $D$.

Proof. Let $C_{a}, C_{a+1}, \ldots$ be the sequence of register configurations that arises from $E$ starting at $C_{a}$. We prove the lemma by induction on the indices of this sequence. The base case $\ell=a$, is trivially true since set $\mathcal{I}_{C_{a}}$ is the empty set.

Suppose that the induction hypothesis is true for $\ell-1 \geq a$. Let the write step between $C_{\ell-1}$ and $C_{\ell}$ be the operation, $w$, by process $p$, into register $\widehat{R}$ with value $V$. Let $s$ be the most recent scan operation by preceding $w$ if it exists.

If $\widehat{S} \subseteq V$, then $\mathcal{T}_{C_{\ell}}=\mathcal{T}_{C_{\ell-1}} \backslash\{\widehat{R}\}$. Define $f_{C_{\ell}}(R)=f_{C_{\ell-1}}(R), \forall R \in \mathcal{T}_{C_{\ell}}$. Since $f_{C_{\ell-1}}$ satisfies the induction hypothesis, and $\mathcal{T}_{C_{\ell}} \subseteq \mathcal{T}_{C_{\ell-1}}, f_{C_{\ell}}$ also satisfies the induction hypothesis.

Now consider the case $\widehat{S} \nsubseteq V$. So $\mathcal{T}_{C_{\ell}}=\mathcal{T}_{C_{\ell-1}} \cup\{\widehat{R}\}$. We first show that $s$ happens before $C_{a}$ or $w$ is the first write by $p$. Suppose, for the purpose of contradiction, that $a \leq \operatorname{index}(s) \leq \ell-1$. We have $\forall R \in \mathcal{R}, \widehat{S} \not \subset \mathrm{v}_{C_{\text {index }(s)}}(R)$ since otherwise, by Line $4, \widehat{S} \subseteq V$. Thus $\left|\mathcal{T}_{C_{\text {index }(s)}}\right|=b$. By the induction hypothesis, $f_{C_{\text {index }(s)}}$ selects a distinct process from each register in $\mathcal{T}_{C_{\text {index }(s)}}$, implying, by Line 4 , that the size of $S_{p}$ is at least $b$. Hence $p$ would have stopped in Line 6 before performing any write operation. Therefore $s$ happens before $C_{a}$ or $w$ is the first write by $p$, and 
consequently any write by $p$ before $w$ happens before $C_{a}$. On the other hand, $\forall R \in \mathcal{T}_{C_{\ell-1}}, f_{C_{\ell-1}}(R)$ performs a write during Interval $[a, \ell-1]$ implying $p$ is not in $\left\{f_{C_{\ell-1}}(R) \mid R \in \mathcal{T}_{C_{\ell-1}}\right\}$. By Observation 5.1, $p \in V$ and $p$ performs a write after $C_{a}$. Therefore by defining $f_{C_{\ell}}(R)=f_{C_{\ell-1}}(R), \forall R \in\left(\mathcal{T}_{C_{\ell-1}} \backslash\{\widehat{R}\}\right)$ and $f_{C_{\ell}}(\widehat{R})=p$, the induction hypothesis holds for $\ell$.

Lemma 5.3. For any execution $E$, let $\widehat{S_{p}}$ and $\widehat{S_{q}}$ be the value of $S_{p}$ and $S_{q}$ in Line 7 for $p$ and $q$ when they have completed the repeat loop. If $\left|\widehat{S_{p}}\right|=\left|\widehat{S_{q}}\right|<b$ then $\widehat{S_{p}}=\widehat{S_{q}}$.

Proof. Let $C_{p}$ and $C_{q}$ be the consistent register configurations that resulted in $\widehat{S_{p}}$ and $\widehat{S_{q}}$ respectively and assume, without loss of generality, that $C_{p}$ precedes $C_{q}$ in $\Gamma_{E}$. By Line $6, R[0]=\cdots=R[b-1]=\widehat{S_{q}}$ in $C_{q}$. Thus, either $\forall R \in R, \widehat{S_{p}} \subseteq v_{C_{q}}(R)$ or $\forall R \in \mathcal{R}, \widehat{S_{p}} \nsubseteq \mathrm{v}_{C_{q}}(R)$.

For the first case, by Line $4, \widehat{S_{p}} \subseteq \widehat{S_{q}}$ and since $\left|\widehat{S_{p}}\right|=\left|\widehat{S_{q}}\right|, \widehat{S_{p}}=\widehat{S_{q}}$. For the latter case, set $\mathcal{T}_{C_{q}}=\left\{R \in \mathcal{R} \mid \widehat{S_{p}} \nsubseteq\right.$ $\left.\vee_{C_{q}}(R)\right\}$ has size $b$. By Lemma 5.2, there is a distinct process in each register in $\mathcal{T}_{C_{q}}$. So there are at least $b$ distinct processes in $\widehat{S_{q}}$ contradicting $\left|\widehat{S_{q}}\right|<b$.

Lemma 5.4. The names returned by any two distinct processes are distinct.

Proof. Let $\widehat{S_{p}}$ and $\widehat{S_{q}}$ be the values of $S_{p}$ and $S_{q}$ in Line7 Without loss of generality, assume that $\left|\widehat{S_{p}}\right| \leq\left|\widehat{S_{q}}\right|$. If $\left|\widehat{S_{p}}\right| \geq b$ and $\left|\widehat{S_{q}}\right| \geq b$, the names returned by $p$ and $q$ in Line 10 are distinct because $p \neq q$. If $\left|\widehat{S_{p}}\right|<b$ and $\left|\widehat{S_{q}}\right| \geq b$, then, by Line 8 , the name returned by $p$ is at most $(b-1)(b-2) / 2+(b-1)=b(b-1) / 2$ and, by Line 10, the name returned by $q$ is bigger than $b(b-1) / 2$. If $\left|\widehat{S_{p}}\right|<b$ and $\left|\widehat{S_{q}}\right|<b$, both processes return at Line 8 . First suppose $\ell=\left|\widehat{S_{p}}\right|<\left|\widehat{S_{q}}\right|$. Then the name returned by $p$ is at most $(\ell+1)(\ell) / 2$ and the name returned by $q$ is at least $(\ell+1)(\ell) / 2+1$. If $\left|\widehat{S_{p}}\right|=\left|\widehat{S_{q}}\right|$, by Lemma 5.3, $\widehat{S_{p}}=\widehat{S_{q}}$. Therefore $\operatorname{rank}\left(p, \widehat{S_{p}}\right) \neq \operatorname{rank}\left(q, \widehat{S_{q}}\right)$. Thus, in all cases the names returned by $p$ and $q$ are distinct.

Observation 5.5. Set $\{p\}$ is written by $p$ before any other write of any set $V \supseteq\{p\}$.

Lemma 5.6. Let $k$ be the number of participating processes during process p's getName (). Then, any name returned by $p$ is in the range $\left\{1, \ldots, \frac{k(k+1)}{2}\right\}$ if $k<b$ and in the range $\left\{1, \ldots, n+\frac{b(b-1)}{2}\right\}$ if $k \geq b$.

Proof. By Observation 5.5, $\forall q \in S_{p}, q$ performs at least one write before $p$ returns. Thus, $\forall q \in S_{p}, q$ is a participating process. Hence, $\left|S_{p}\right| \leq k$. If $k<b$, then $\left|S_{p}\right|<b$. Therefore, process $p$ returns in Line 8 and the name is in the range $\left\{1, \ldots, \frac{k(k+1)}{2}\right\}$. If $k \geq b$, then $p$ returns either in Line 8 or in Line 10 . Therefore the name is in the range $\left\{1, \ldots, \frac{b(b-1)}{2}+n\right\}$.

In summary, Lemmas 5.4 and 5.6 imply that the algorithm in Fig. 1 is an $(b-1)$-bounded $(k(k+1) / 2)$-adaptive renaming algorithm that uses $b$ registers assuming the availability of the atomic scan operation.

\section{Step complexity.}

We now bound the maximum number of steps (scans and writes) that any process can take during its execution of getName. Lemma 5.7 establishes the most important piece of the step complexity of the algorithm in Fig. 1 In this lemma we prove that if there exists a register configuration in which there are at least $k$ registers, each of which contains a set of size at least $k$, then the number of distinct process names in any subsequent scan is at least $k$. We call such a register configuration $k$-complete, and any set of such registers is a $k$-full-set. The core idea is that after a $k$-complete configuration with $k$-full-set $\mathcal{R}^{\prime}$, every write with set size less than $k$ to a register in $\mathcal{R}^{\prime}$ is performed by a distinct writer. It then follows that the union of the sets appearing in $\mathcal{R}^{\prime}$ always will have size at least $k$. For the proof, given set of registers $\mathcal{R}^{\prime} \subseteq \mathcal{R}$ and a register configuration $D$, we will be interested in those registers in $\mathcal{R}^{\prime}$ that contain a set smaller than $k$, and in the processes that wrote these small sets to these registers. Therefore, define $\rho_{\mathcal{R}^{\prime}}(D, k)=\left\{R \in \mathcal{R}^{\prime}|| v_{D}(R) \mid<k\right\}$. Let writer $(j, R)$ denote the process that performs the most recent write to register $R$ preceding register configuration $C_{j}$. For any set of registers $\mathcal{R}^{\prime}$, register configuration $C_{j}$ and an integer $k$, define $W_{\mathcal{R}^{\prime}}(j, k)=\left\{\operatorname{writer}(j, R) \mid R \in \rho_{\mathcal{R}^{\prime}}\left(C_{j}, k\right)\right\}$. Notice that a register configuration $D$ is $k$-complete if there exists a set $\mathcal{R}^{\prime}$ of $k$ registers where $\rho_{\mathcal{R}^{\prime}}(D, k)=\emptyset$. Furthermore, $\mathcal{R}^{\prime}$ is $k$-full-set at register configuration $D$.

Lemma 5.7 uses a proof structure that is more elaborate than, but reminiscent of, that of Lemma 5.2 . 
Lemma 5.7. For any execution $E$, let $C_{a}$ be a $k$-complete register configuration where $0 \leq k \leq b-1$ and let $\mathcal{R}^{\prime}$ be a $k$-full-set of $C_{a}$. For any register configuration $C_{e}$ following $C_{a}$ in $\Gamma_{E}$, there exists a one-to-one and onto function $g_{C_{e}}: \rho_{\mathcal{R}^{\prime}}\left(C_{e}, k\right) \rightarrow W_{\mathcal{R}^{\prime}}(e, k)$ satisfying, $\forall R \in \rho_{\mathcal{R}^{\prime}}\left(C_{e}, k\right), g_{C_{e}}(R) \in v_{C_{e}}(R)$. Furthermore, each process in $W_{\mathcal{R}^{\prime}}(e, k)$ performs at least one write in Interval $[a, e]$.

Proof. Let $C_{a}, C_{a+1}, \ldots$ be the sequence of all register configurations starting at $C_{a}$. We prove the lemma by induction on the indices of this sequence. The base case $\ell=a$, is trivially true since set $\rho_{\mathcal{R}^{\prime}}\left(C_{a}, k\right)$ is the empty set.

Suppose that the induction hypothesis is true for $\ell-1 \geq a$. Let the write step between $C_{\ell-1}$ and $C_{\ell}$ be the operation, $w$, by process $q$, into register $\widehat{R}$ with value $V$. Let $s$ be the most recent scan operation by $q$ preceding $w$ if it exists.

Suppose that $\widehat{R} \notin \mathcal{R}^{\prime}$. Then $\rho_{\mathcal{R}^{\prime}}\left(C_{\ell-1}, k\right)=\rho_{\mathcal{R}^{\prime}}\left(C_{\ell}, k\right)$ and $W_{\mathcal{R}^{\prime}}(\ell-1, k)=W_{\mathcal{R}^{\prime}}(\ell, k)$, so the induction hypothesis holds trivially for $\ell$ by setting $g_{C_{\ell}}=g_{C_{\ell-1}}$.

Suppose that $\widehat{R} \in \mathcal{R}^{\prime}$ and $|V| \geq k$. Then $\rho_{\mathcal{R}^{\prime}}\left(C_{\ell}, k\right)=\rho_{\mathcal{R}^{\prime}}\left(C_{\ell-1}, k\right) \backslash\{\widehat{R}\}$ and $W_{\mathcal{R}^{\prime}}(\ell, k)=W_{\mathcal{R}^{\prime}}(\ell-1, k) \backslash$ $\{\operatorname{writer}(\ell-1, \widehat{R})\}$. So the hypothesis holds for $\ell$ by setting $g_{C_{\ell}}=g_{C_{\ell-1}}$ for each $R \in \rho_{\mathcal{R}^{\prime}}\left(C_{\ell}, k\right)$.

Finally, consider the case $\widehat{R} \in \mathcal{R}^{\prime}$ and $|V|<k$. We first show that $s$ happens before $C_{a}$ or $w$ is the first write by $q$. Suppose, for the purpose of contradiction, that $s$ happens after $C_{a}$. Then $a \leq \operatorname{index}(s) \leq \ell-1$. For each $R \in \mathcal{R}^{\prime}$, we have $\left|v_{C_{\text {index }(s)}}(R)\right|<k$ since otherwise, by Line $4|V| \geq k$. Thus $\left|\rho_{\mathcal{R}^{\prime}}\left(C_{\text {index }(s)}, k\right)\right|=k$. By the induction hypothesis, $g_{C_{\text {index }(s)}}$ is a bijection, so $\left|\rho_{\mathcal{R}^{\prime}}\left(C_{\text {index }(s)}, k\right)\right|=\left|W_{\mathcal{R}^{\prime}}(\operatorname{index}(s), k)\right|=k$, and $\forall R \in \rho_{\mathcal{R}^{\prime}}\left(C_{\text {index }(s)}, k\right), g_{C_{\text {index }(s)}}(R) \in v_{C_{\text {index }(s)}}(R)$. Therefore, by Line 4, the size of $S_{q}$, and hence the size of $V$, is at least $k$, which is a contradiction.

Therefore $s$ happens before $C_{a}$ or $w$ is the first write by $q$, and consequently any write by $q$ before $w$ happens before $C_{a}$. On the other hand, by the induction hypothesis, $\forall R \in \rho_{\mathcal{R}^{\prime}}\left(C_{\ell-1}, k\right)$, writer $(\ell-1, R)$ performs a write during Interval $[a, \ell-1]$ implying $q$ is not in $W_{\mathcal{R}^{\prime}}(\ell-1, k)$. We have $\rho_{\mathcal{R}^{\prime}}\left(C_{\ell}, k\right)=\rho_{\mathcal{R}^{\prime}}\left(C_{\ell-1}, k\right) \cup\{\widehat{R}\}$ and $W_{\mathcal{R}^{\prime}}(\ell, k)=$ $W_{\mathcal{R}^{\prime}}(\ell-1, k) \backslash$ writer $(\ell-1, \widehat{R}) \cup\{q\}$, whether or not $\widehat{R}$ is in $\rho_{\mathcal{R}^{\prime}}\left(C_{\ell-1}, k\right)$. Furthermore, $q$ performs a write after $C_{a}$. Therefore, the induction hypothesis holds for $\ell$ by defining $g_{C_{\ell}}=g_{C_{\ell-1}}$ for each $R \in \rho_{\mathcal{R}^{\prime}}\left(C_{\ell}, k\right) \backslash\{\widehat{R}\}$, and $g_{C_{\ell}}(\widehat{R})=q$.

Lemma 5.8. Let $D$ be a $k$-complete register configuration. Then for each process $p$ in $\mathcal{P}$, $p$ 's second write after $D$ if it exists, has a value with size at least $k$.

Proof. Let $w$ be the second write operation by $p$ after $D$ if it exists. Let $s$ be the most recent scan operation by $p$ preceding $w$. Since $w$ is the second write by $p$, the value returned by $s$ is equal to a register configuration $D^{\prime}$ following $D$. Let $\mathcal{R}^{\prime}$ be the $k$-full-set of $D$. If $\exists R \in \mathcal{R},\left|v_{D^{\prime}}(R)\right| \geq k$, then by Line 4 , the size of $S_{p}$ at $s$ is at least $k$. Otherwise, all registers, and hence all registers in $\mathcal{R}^{\prime}$, contain sets of size less than $k$. Therefore, $\left|\rho_{\mathcal{R}^{\prime}}\left(D^{\prime}, k\right)\right|=k$. So, by Lemma 5.7, $\mid W_{\mathbb{R}^{\prime}}\left(\right.$ index $\left.\left(D^{\prime}\right), k\right) \mid=k$. Thus, again by Line 4, the size of $S_{p}$ at $s$ is at least $k$. Hence $w$ has a value with size at least $k$.

Lemma 5.9. Let $E$ be an execution whose first operation is a write by $p$ and contains the next $b$ scans by $p$. Furthermore, during $E$, every write by $p$ has value $Q$ and no write has value $Q^{\prime} \subsetneq Q$. Then $p$ either terminates or the size of $S_{p}$ increases.

Proof. By Lines 2, 3, each scan operation is preceded by a write operation. Hence $E$ contains $b$ writes by $p$. Therefore, during $E, p$ writes $Q$ to all $b$ registers. Let $\left(V_{0}, \ldots, V_{b-1}\right)$ be the value returned by $p$ 's last scan during $E$. Because $E$ does not contain any write with value $Q^{\prime} \subsetneq Q$ either $V_{0}=\cdots=V_{b-1}=Q$ in which case $p$ terminates or $\exists i, 0 \leq i \leq b-1$ such that $V_{i} \nsubseteq Q$. In the latter case by Line 4 , the size of $S_{p}$ increases.

Lemma 5.10. Let $D$ be a $k$-complete register configuration where $0 \leq k \leq b-1$. Then for each process $p$ in $\mathcal{P}$, $p$ 's $(b k+2)$-nd write after $D$, if it exists, has a value with size at least $k+1$.

Proof. Let $w$ be the second write by $p$ after $D$. Suppose that $p$ writes $Q$ at $w$. By Lemma $5.8,|Q| \geq k$. If $|Q| \geq k+1$ or $p$ terminates before writing $b k$ more times, we are done. Therefore, suppose that $|Q|=k$ and $p$ performs $b k$ writes after $w$. Then $|Q \backslash\{p\}|=k-1$. By Lemma 5.8, after $D, \forall q \in Q, q$ writes a value with size smaller than $k$ at most once. 
Let $E$ be the execution whose first operation is $w$ and contains the next $b k$ scan operations by $p$. Partition $E$ into disjoint segments, $E=\left(E_{1}, \ldots, E_{k}\right)$, satisfying $\forall \ell, 1 \leq \ell \leq k$, the first operation in $E_{\ell}$ is a write operation by $p$ and $E_{\ell}$ contains the next $b$ scans by $p$. Notice that $E$ contains exactly $b k$ write operations by $p$ and since $w$ is the first operation of $E, p$ performs at least one more write after $E$ ends. Since there are at most $|Q \backslash\{p\}|=k-1$ writes after $w$ that have a value $V$ satisfying $V \subsetneq Q$, there exists an $\ell, 1 \leq \ell \leq k$ such that all writes during $E_{\ell}$ have a value that is not a proper subset of $Q$. Since $p$ does not terminate during $E_{\ell}$, by Lemma 5.9, the size of $S_{p}$ after $E_{\ell}$ (hence, after $E$ ) is at least $k+1$. Hence, $p$ 's $(b k+2)$-nd write after $D$ has a value with size at least $k+1$.

Lemma 5.11. For any execution $E$ in which $p$ does not terminate, let $O$ be the set of all scan operations by $p$ during E. Let $Z=\{$ writer $(\operatorname{index}(s), R) \mid s \in O$ and $R \in \mathcal{R}\}$. Then $|Z|<b$.

Proof. For any scan $s \in O$ and any register $R$, writer $(\operatorname{index}(s), R) \in \mathrm{v}_{C_{\text {index }(s)}}(R)$. Therefore, by Line 4 for any $s \in O$, after $s, S_{p}$ contains writer $(\operatorname{index}(s), R)$. Since $p$ does not terminate after $s$, at $s,\left|S_{p}\right|<b$. Hence $|Z|<b$.

Lemma 5.12. Let $D$ be a $k$-complete register configuration where $0 \leq k \leq b-1$. Then for each process $p$ in $\mathcal{P}$, $p$ makes at most $b k+1+b\left(\frac{(b-1)(b k+1)}{b-k}+1\right)$ write operations before it terminates or a $(k+1)$-complete register configuration is achieved.

Proof. By Lemma[5.11 $p$ 's $(b k+2)$-nd write, say $w$, after $D$ has a value with size at least $k+1$. Let $E$ be the execution whose first operation is $w$ and contains the next $b\left(\frac{(b-1)(b k+1)}{b-k}+1\right)$ scan operations by $p$. Partition $E$ into segments, $E=$ $\left(E_{1}, \ldots, E_{\frac{(b-1)(b k+1)}{b-k}+1}\right)$, satisfying $\forall \ell, 1 \leq \ell \leq \frac{(b-1)(b k+1)}{b-k}+1$, the first operation in $E_{\ell}$ is a write operation by $p$ and $E_{\ell}$ contains the next $b$ scans by $p$. Let $O$ be the set of all scan operations by $p \operatorname{during} E$. Let $Z=\{\operatorname{writer}(\operatorname{index}(s), R) \mid s \in$ $O$ and $R \in R$ R. Let $U$ be the set of all write operations by processes in $Z$ during $E$ such that $\forall u \in U$, the value of $u$ has a size smaller than or equal to $k$. By Lemmas 5.10 and 5.11, $|U| \leq(b k+1)|Z| \leq(b-1)(b k+1)$. Let $U_{\ell}=\left\{u \mid u \in U\right.$ and $u$ happens during $\left.E_{\ell}\right\}$.

By the pigeon whole principle, there exists an $\ell$ such that $\left|U_{\ell}\right|<b-k$. Let $s_{\ell}$ be $p$ 's last scan during $E_{\ell}$. Since during $E_{\ell}, p$ writes a value with size at least $k+1$ to all $b$ registers and the number of writes with value smaller than $k+1$ and scanned by $p$ (i.e $\left|U_{\ell}\right|$ ), is less than $b-k, s_{\ell}$ returns a view in which at least $k+1$ registers have size at least $k+1$. Hence, $C_{\text {index }\left(s_{\ell}\right)}$ is $(k+1)$-complete.

Lemma 5.13. No process writes more than $3 b^{4} \ln b$ times.

Proof. By Lemma 5.12, a process can write at most $b k+1+b\left(\frac{(b-1)(b k+1)}{b-k}+1\right)$ times between a $k$-complete and a $(k+1)$-complete configuration. The initial configuration is 0 -complete and an $b$-complete configuration cannot exist. Therefore a process can write a most

$$
\begin{aligned}
& \sum_{k=0}^{b-1}\left(b k+1+b\left(\frac{(b-1)(b k+1)}{b-k}+1\right)\right) \\
= & \sum_{k=0}^{b-1}(b k+1+b)+b(b-1) \sum_{k=0}^{b-1}\left(\frac{b k+1}{b-k}\right) \\
= & \sum_{k=0}^{b-1}(b k+1+b)+b(b-1) \sum_{k=1}^{b}\left(\frac{b^{2}-b k+1}{k}\right) \\
< & \frac{b^{3}}{2}+b-1+b^{2}+b^{2} \sum_{k=1}^{b}\left(\frac{b^{2}}{k}-b+\frac{1}{k}\right)<3 b^{4} \ln b
\end{aligned}
$$

times before it terminates. 
We replace the atomic scan in Fig. 1 with a new function, newScan (), and the getName () algorithm also changes accordingly. The revised renaming algorithm is shown in Fig. 2 In the getName () algorithm, processes augment the values they write to each register with their ids and sequence numbers in order to guarantee the uniqueness of the value of each write. This prevents the ABA problem. Each register $R \in \mathcal{R}$ stores an ordered triple (set, id, seqNumber).

During a newScan () operation by process $p, p$ performs a collect $(\mathcal{R})$ in Line 18 , by reading $R[0]$ through $R[b-1]$ consecutively and returns a collect. After each collect $(\mathcal{R}), p$ updates its set $S$ from this collect. It repeatedly gets a collect until either the size of set $S$ becomes at least $b$ or it obtains two identical consecutive collects and returns this collect. If newScan () terminates at Line 24, then the returned collect is equivalent to the returned value of a linearizable implementation of a scan [21, 26, 25, 14, 15]. Hence all the proofs in Section 5.1]hold when newScan () terminates at Line 24. So to establish the correctness, we need to prove that when a process $p$ returns in Line 21, in fact more than $b-1$ processes are participating, hence the name returned by p's getName () is valid. This is shown in Lemma 5.14. In Lemma 5.13, we showed that the number of writes by each process is bounded. Since the sequence number seqNumber, cannot get larger than the number of write operations by each process, the size of each register is also bounded. Therefore, after Lemma 5.14 it will remain to prove that the getName () algorithm in Fig. 22 is wait-free. This will be established in Lemmas 5.15 through 5.17, by bounding the number of steps of each newScan () operation.

For any read operation $o$ of register $R$, define writeOp $(o)$ to be the most recent write operation to $R$ preceding $o$ if it exists and $\perp$ otherwise. For any write operation $w$, let performer $(w)$ denote the process that performs $w$. For any set of write operations $W$, let $Z(W)=\{\operatorname{performer}(w) \mid w \in W\}$.

Lemma 5.14. Let $k$ be the number of participating processes during process p's getName (). If a newScan () operation by $p$ returns in Line 21, then $k \geq b$.

Proof. Let $\widehat{S_{p}}$ be the value of $S_{p}$ when $p$ 's getName () returns. Since $p$ 's newScan () operation returns in Line 21 $\left|\widehat{S_{p}}\right| \geq b$. By Observation 5.5, $\forall q \in \widehat{S_{p}}, q$ performs at least one write before $p$ returns. Thus, $\forall q \in \widehat{S_{p}}, q$ has invoked a getName () before $p$ returns. Therefore, $k \geq\left|\widehat{S_{p}}\right| \geq b$.

Lemma 5.15. Let $E$ be an execution such that any step by process $p$ during $E$ is part of a single newScan () operation. If $E$ contains at least $3 b$ reads by $p$ and does not contain any write operations, then p's newScan () terminates during $E$.

Proof. Since $E$ contains no write operation, every $3 b$ reads by $p$ must contain two complete identical collects. Hence, $p$ must terminate due to Line 23.

Lemma 5.16. Let $E$ be an execution such that any step by process $p$ during $E$ is part of a single newScan () operation, s. Let $O$ be the set of all reads by $p$ during $E$ and $W=\{$ writeOp $(o) \mid o \in O\} \backslash\{\perp\}$. If $|Z(W)| \geq b$, then s contains at most $2 b$ read operations after $E$ ends.

Proof. If $p$ performs fewer than $2 b$ read operations after $E$ ends, we are done. Let $E^{\prime}$ be an execution which starts after $E$ ends and contains $2 b$ reads by $p$. Since every $2 b$ reads by $p$ must contain a complete collect, after $p$ 's complete collect during $E^{\prime}$, by Line 19, $S_{p}$ includes all processes in $Z(W)$. Hence, after $p$ 's complete collect during $E^{\prime}$, $\left|S_{p}\right| \geq|Z(W)| \geq b$. Therefore, by Line 20, s must terminate.

Lemma 5.17. No newScan () operation contains more than $10 b^{6} \ln b$ reads.

Proof. By way of contradiction, let $E$ be an execution in which process $p$ performs a single newScan () $s$, and it contains more than $10 b^{6} \ln b$ reads. Let $E^{\prime}$ be a prefix of $E$ that contains $9 b^{6} \ln b$ reads by $p$. Partition $E^{\prime}$ into disjoint segments, $E^{\prime}=\left(E_{1}, \ldots, E_{3 b^{5} \ln b}\right)$, satisfying $\forall \ell, 1 \leq \ell \leq 3 b^{5} \ln b, E_{\ell}$ contains $3 b$ reads by $p$. Let $O$ be the set of all read operations by $p$ during $E^{\prime}$ and $W=\{$ writeOp $(o) \mid o \in O\} \backslash\{\perp\}$.

Suppose there is an $\ell$ such that $E_{\ell}$ contains no write operation in $W$. This implies that $E_{\ell}$ contains no write operation. Therefore by Lemma 5.15, $p$ terminates $s$ during $E_{\ell}$. 
shared: $R[0 \ldots b-1]$ is an array of multi-writer multi-reader registers, each register is initialized to $(\emptyset, 0,0)$; local: $r[0, \ldots, b-1]$; pos $\in\{0, \ldots, b-1\}$ initialized to 0 ; seqNumber is a non-negative integer initialized to $0 ; S$ is initialized to $\{$ id $\}$; largeSet is a boolean;

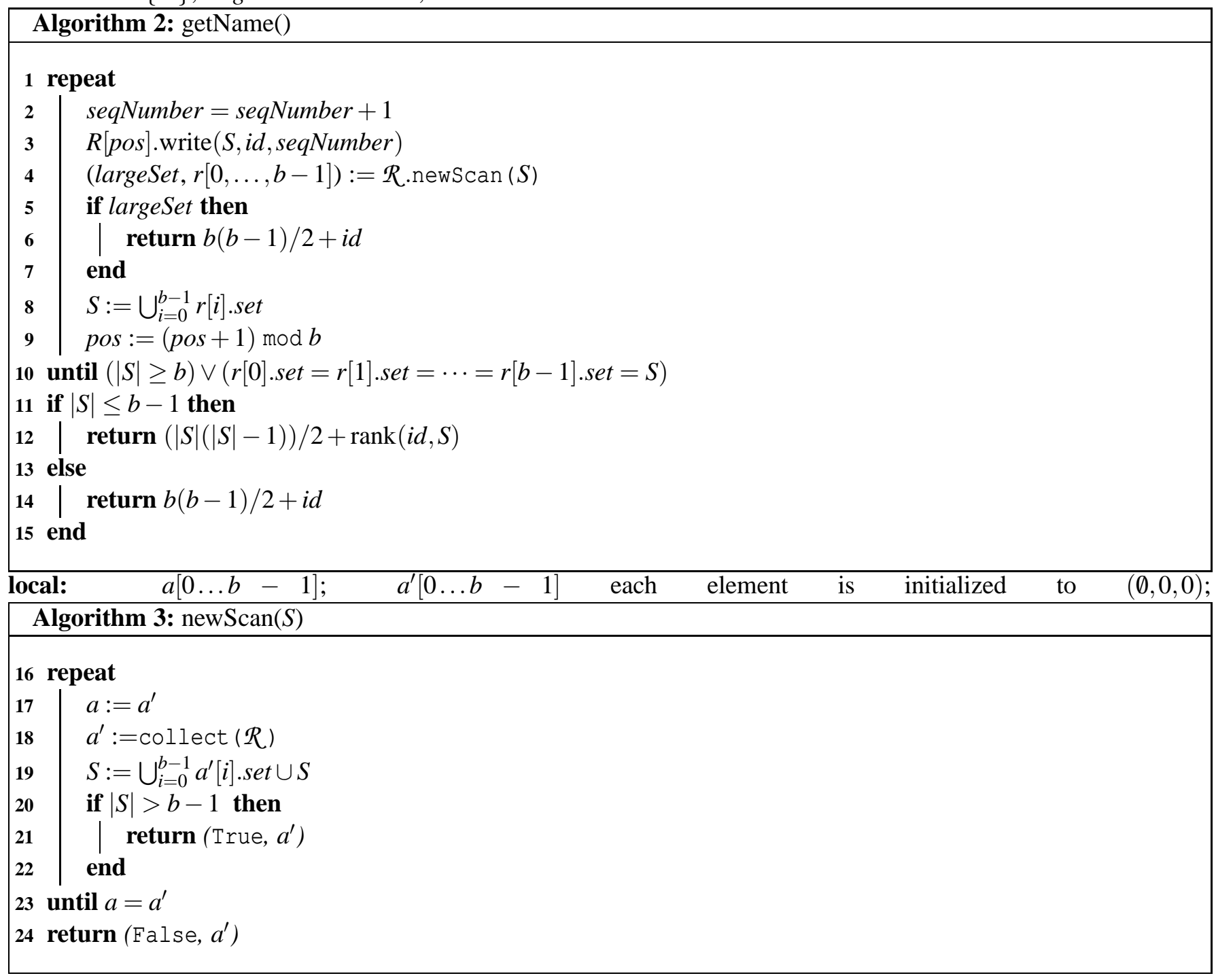

Figure 2: $(b-1)$-Bounded $(k(k+1) / 2)$-Adaptive Renaming Using Registers 
Otherwise, each $E_{\ell}$ contains at least one write in $W$. Hence $|W| \geq 3 b^{5} \ln b$. Since by Lemma 5.13, each process $p$ performs at most $3 b^{4} \ln b$ writes, $|Z(W)| \geq b$. Therefore, by Lemma 5.16, $s$ contains at most $2 b$ reads after $E^{\prime}$ ends. Hence $E$ contains at most $9 b^{6} \ln b+2 b<10 b^{6} \ln b$ reads by $p$.

Lemma 5.18. No process performs more than $31 b^{10} \ln ^{2} b$ shared steps (read or write).

Proof. By Lemma 5.13, each process $p$ performs at most $3 b^{4} \ln b$ writes. Hence, $p$ performs at most $3 b^{4} \ln b$ newScan () operations. By Lemma 5.17, p performs at most $10 b^{6} \ln b$ reads in each newScan () operation. Hence $p$ performs at most $3 b^{4} \ln b+\left(3 b^{4} \ln b\right)\left(10 b^{6} \ln b\right) \leq 31 b^{10} \ln ^{2} b$ shared steps.

Theorem 5.19. For any $b \geq 2$, there is a wait-free one-shot $(b-1)$-bounded $(k(k+1) / 2)$-adaptive renaming algorithm implemented from $b$ bounded registers. Additionally, when $k \geq b$, the returned names are in the range $\left\{1, \ldots, n+\frac{b(b-1)}{2}\right\}$.

Setting $b=\lceil\sqrt{n}\rceil+1$, we have a wait-free one-shot $\lceil\sqrt{n}\rceil$-bounded $(k(k+1) / 2)$-adaptive renaming algorithm from $\lceil\sqrt{n}\rceil+1$ bounded registers. This implies that the algorithm returns names in the range $\{1, \ldots,(k(k+1) / 2)\}$ when $k \leq\lceil\sqrt{n}\rceil$, and returns names in the range $\left\{1, \ldots, n+\frac{\lceil\sqrt{n}\rceil(\lceil\sqrt{n}\rceil+1)}{2}\right\}$ when $k \geq\lceil\sqrt{n}\rceil+1$. Note that when $k \geq\lceil\sqrt{n}\rceil+1$, $n+\frac{[\sqrt{n}]([\sqrt{n}]+1)}{2} \leq k^{2}+\frac{k^{2}}{2}$. Hence, $\forall k \in\{1, \ldots, n\}$, the algorithm returns names in the range $\left\{1, \ldots,\left(3 k^{2}\right) / 2\right\}$.

Corollary 5.1. There is a wait-free one-shot $\left.\left(3 k^{2}\right) / 2\right)$-adaptive renaming algorithm implemented from $\lceil\sqrt{n}\rceil+1$ bounded registers.

\section{Obstruction-Free $(b-1)$-Bounded $k$-Adaptive Renaming}

Fig. 3 presents pseudo-code for an obstruction-free one-shot $(b-1)$-bounded $k$-adaptive renaming algorithm from $b$ registers assuming an atomic scan operation. In Theorem 6.7 we show how to remove this assumption by adding an extra register.

\section{Algorithm Description.}

A naming set is a set of ordered pairs where each pair is a process id and a proposed name with the property that no process id occurs in more than one pair in the set. Let $S$ be a naming set. In our algorithm and the analysis we use the following notation:

- $\operatorname{Procs}(S)=\{x \mid(x, y) \in S\}$,

- $\operatorname{Names}(S)=\{y \mid(x, y) \in S\}$,

- if $(p, n) \in S$, then name $(p, S)$ is $n$; otherwise it is undefined.

The algorithm in Fig. 3 employ a set $\mathcal{R}=\{R[1], \ldots, R[b]\}$ of shared atomic registers. Each register $R$ stores an ordered triple (set,writer,proposal) where set is a naming set, writer is a process id or $\perp$ (initially) and proposal is a positive integer less than or equal $b-1$. Each process $p$ maintains a naming set $S_{p}$ and alternates between write and scan operations until it terminates with a name for itself. Each scan returns a view, which is an atomic snapshot of the content of all registers. Each write by $p$ writes a triple consisting of its set $S_{p}$, its $i d p$, and its proposed name name $e_{p}$, to some register $R[j]$. Process $p$ uses its last view and its previous value of $S_{p}$ to determine the new value of $S_{p}$, name and $j$.

Function Update describes how $p$ constructs $S_{p}$ in three steps. In the first step (Lines 18, 22), $p$ creates a naming set based only on the writers and proposals of each register in its view. If the view contains a writer with more than one proposal, $p$ chooses one pair arbitrarily. In the second step (Lines 23 31), $p$ augments its naming set with additional pairs for processes that are not writers in its view but occur in the union of all naming sets in its view. The main issue occurs when there is some process that is paired with more than one name from two or more naming sets in different registers. In this case, if there are two such registers with the same writer then, $p$ chooses the pair which occurs in the register with bigger index. Otherwise, $p$ picks one pair arbitrarily. Finally (Lines 32 34), $p$ adds any pair $\left(q, n_{q}\right)$ such that $q$ exists in the previous version of $S_{p}$ and is not yet added. Observe that $S_{p}$ is a naming set and $p \notin \operatorname{Procs}\left(S_{p}\right)$.

In Line $5 p$ chooses its proposal for its own name, name ${ }_{p}$, to be the smallest integer that is not paired with some other process in $S_{p}$. 
shared: $\mathcal{R}=R[1, \ldots, b]$ is an array of multi-writer multi-reader registers, each register is initialized to $(\emptyset, \perp, 1)$ local: $r[1, \ldots, b]$; pos $\in\{1, \ldots, b\}$ initialized to $1 ; S$ initialized to $\emptyset$; proposed $\in \mathbb{N}$ initialized to 1 .

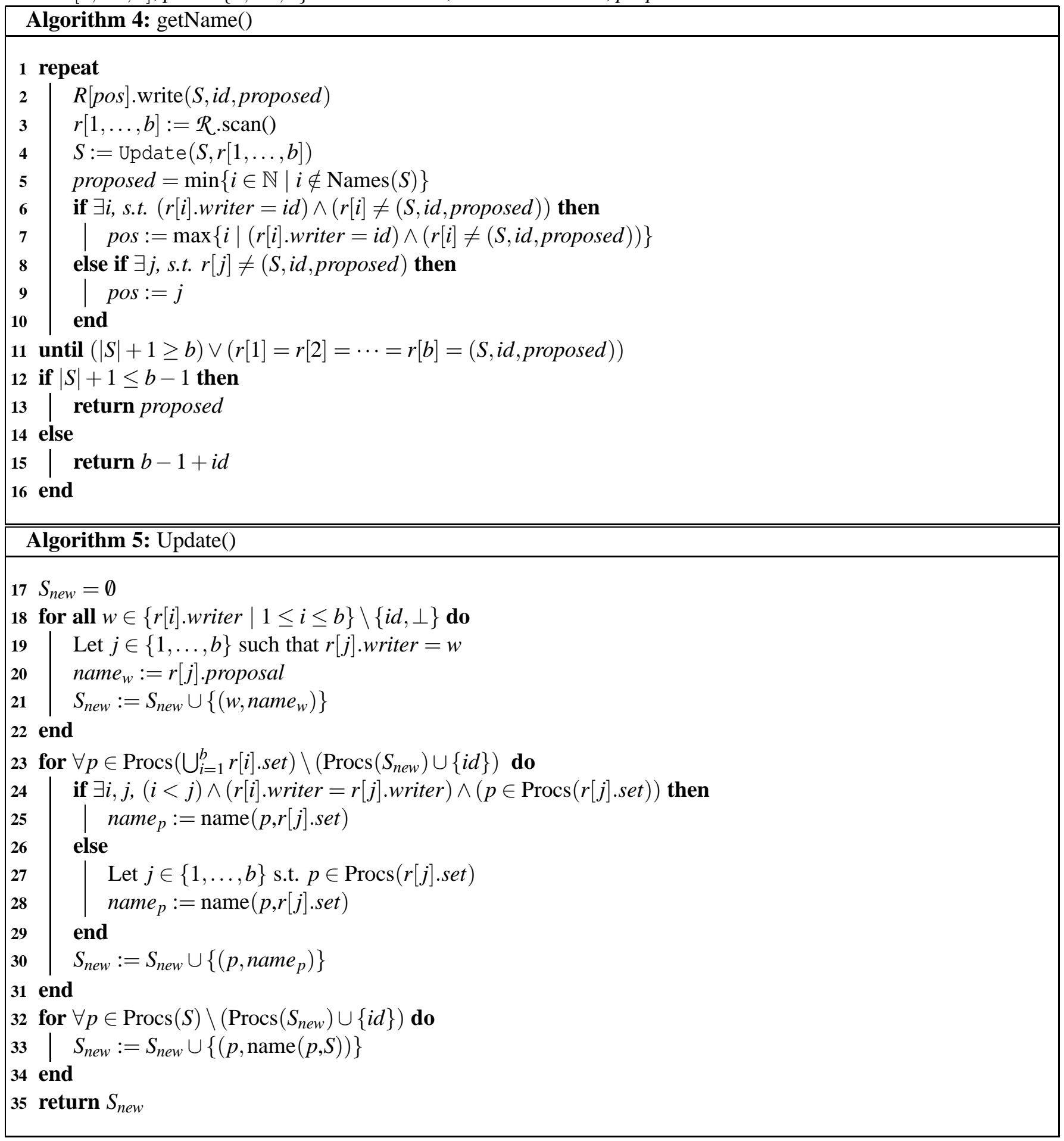

Figure 3: $(b-1)$-Bounded $k$-Adaptive Renaming 
Lines 6 10 describe how $p$ sets $j$. If there is any register in $p$ 's preceeding view with writer component equal to $p$ but with content different from $\left(S_{p}, p\right.$, name $\left._{p}\right)$ then $p$ writes to register $R[j]$ where $j$ is the biggest index amongst these registers. Otherwise it writes to some register whose content is different than $\left(S_{p}, p\right.$, name $\left._{p}\right)$. Process $p$ continues until either in some scan, all registers contain the same information that $p$ has written or $\left|S_{p}\right|$ is larger than or equal $b-1$. In the first case $p$ returns name $_{p}$ and in the second case it returns $b+p-1$.

\section{Proof of Correctness}

Overview of proof Once a process $p$ terminates with name $n_{p} \leq b-1$, the pair (writer, proposal) of every register is equal to $\left(p, n_{p}\right)$. The core idea is that after $p$ terminates, every register that is overwritten with the wrong name for $p$ or no name for $p$, has a distinct writer component. Therefore, if a subsequent scan by another process, say $q$, does not include the correct name for $p$, the set of processes in that scan is large and $q$ terminates with a name larger than or equal $b$. If the set of processes in the scan is not large, then there is some writer that is in the writer component of at least 2 registers. In that case, we prove that for any such pair of registers with the same writer, the correct name for $p$ is in the register with the larger index. In this way, the algorithm ensures that process $q$ keeps $\left(p, n_{p}\right)$ in its naming set, and discards incorrect names for $p$.

For our proof, we use the notion and terminology for register configuration, consistent configuration, index $(o p)$ of operation $o p$, interval and content of register $R$ in configuration $C, v_{C}(R)$, as defined in Section 5 Let $p$ be a process that has terminated and returned name $n_{p}$. Define last $p$ to be the last scan by $p$. For any register configuration $D$ following register configuration $C_{\text {index (last }}$ ), define a set of registers $\mathcal{F}_{p}(D)=\left\{R \in \mathcal{R} \mid\left(v_{D}(R)\right.\right.$. writer $\left.\neq p\right) \wedge\left(\left(p, n_{p}\right) \notin\right.$ $v_{D}(R)$.set $\left.)\right\}$ and a set of processes $O_{p}(D)=\bigcup_{R \in \mathcal{F}_{p}(D)}\left\{\mathrm{v}_{D}(R)\right.$.writer $\}$.

Lemma 6.1. Let $E$ be any execution starting in the initial configuration and ending in configuration $C$. If there are two integers $i$ and $j$ such that $i<j, v_{C}(R[i])$.writer $=v_{C}(R[j])$.writer $=p$ and $v_{C}(R[i]) \neq v_{C}(R[j])$, then the last write to $R[i]$ happens before the last write to $R[j]$ and both are by the same process.

Proof. By Line 2, the writer segment of each register indicates the id of the process which writes that value. Hence, $v_{C}(R[i])$ and $v_{C}(R[j])$ are both written by the same process $p$. Let $w_{i}$ and $w_{j}$ be the most recent writes to $R[i]$ and $R[j]$ preceding $C$, respectively. Thus, value of $w_{i}$ (respectively $w_{j}$ ) is $v_{C}(R[i])$ (respectively $v_{C}(R[j])$ ). By way of contradiction assume that $w_{j}$ happens before $w_{i}$. Let $s_{i}$ be the most recent scan operation by $p$ before $w_{i}$. Hence $s_{i}$ happens after $w_{j}$ and before $w_{i}$. Since $w_{j}$ is the most recent write to $R[j]$ preceding $s_{i}, v_{C_{\text {index }\left(s_{i}\right)}}(R[j])=v_{C}(R[j])$. Let

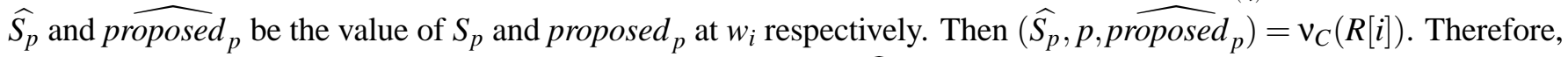
when $p$ executed Line 6 preceding $w_{i}$ and after $s_{i}, \widehat{S_{p}}$ and proposed $d_{p}$ are values of $S_{p}$ and proposed ${ }_{p}$ respectively.

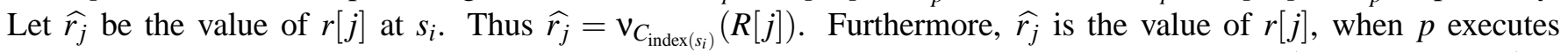
Line 6 after $s_{i}$ and preceding $w_{i}$. Therefore at the execution of Line 6 after $s_{i}$ and preceding $w_{i},\left(S_{p}, p\right.$, proposed $\left._{p}\right)=$ $\left(\widehat{S_{p}}, p, \widehat{\text { proposed }}_{p}\right)=\mathrm{v}_{C}(R[i]) \neq \mathrm{v}_{C}(R[j])=\mathrm{v}_{C_{\text {index }\left(s_{i}\right)}}(R[j])=\widehat{r_{j}}=r[j]$ and $r[j] . i d=\widehat{r_{j}} . i d=p$. Thus, Line 6 evaluates to true. Since $j>i$, by Line $7, p$ does not write into $R[i]$ before writing into $R[j]$.

Informally, Lemma 6.2 says that every register that contains an incorrect name for $p$ after a consistent configuration containing the correct name for $p$ has a distinct writer component.

Lemma 6.2. Consider an execution $E$ in which process $p$ 's getName () call returns name $n_{p} \leq b-1$. Then for any register configuration $C_{e}$ where $e \geq \operatorname{index}\left(\right.$ last $\left._{p}\right)$,

i) $\left|\mathcal{F}_{p}\left(C_{e}\right)\right|=\left|O_{p}\left(C_{e}\right)\right|$;

ii) $\forall q \in O_{p}\left(C_{e}\right)$, q performs a write in Interval [index $\left(\right.$ last $\left.\left._{p}\right), e\right]$; and

iii) for any write operation o by any process $q$ during Interval [index $\left(\right.$ last $\left.\left._{p}\right), e\right]$, let $v$ be the value of $o$. If o is not $q$ 's first write during Interval $\left[\right.$ index $\left(\right.$ last $\left.\left._{p}\right), e\right]$, then $\left(p, n_{p}\right) \in$ v.set.

Proof. Let $C_{\mathrm{index}\left(\text { last }_{p}\right)}, C_{\mathrm{index}\left(\text { last }_{p}\right)+1}, \ldots$ be the sequence of all register configurations starting at $C_{\mathrm{index}\left(\text { last }_{p}\right)}$. We prove the lemma by induction on the indices of this sequence. Let $\widehat{S_{p}}$ be the value of $S_{p}$ at last $t_{p}$. For the base case $\ell=\operatorname{index}\left(\right.$ last $\left._{p}\right)$, since $n_{p} \leq b-1, p$ returns in Line 13 Therefore the condition $r[1]=\cdots=r[b]=\left(\widehat{S_{p}}, p, n_{p}\right)$ 
held when $p$ last executed Line 11. Hence, condition $R[1]=\cdots=R[b]=\left(\widehat{S_{p}}, p, n_{p}\right)$ held at $\left.C_{\text {index }(\text { last }}\right)$. Therefore the induction hypothesis $(i)$ and $(i i)$ hold for the base case $\ell=\operatorname{index}\left(\right.$ last $\left._{p}\right)$ because $\mathcal{F}_{p}\left(C_{\text {index }\left(\text { last }_{p}\right)}\right)=O_{p}\left(C_{\text {index }\left(\text { last }_{p}\right)}\right)=\emptyset$. Furthermore, since Interval [index $\left(\right.$ last $\left._{p}\right)$, index $\left(\right.$ last $\left.\left._{p}\right)\right]$ contains only one write, (iii) is true for the base case.

Suppose that the lemma holds for $\ell-1 \geq \operatorname{index}\left(\right.$ last $\left._{p}\right)$. Let $w$ be the write that changes register configuration $C_{\ell-1}$ to $C_{\ell}$, and let $x$ be the process that performs $w$. Then clearly $x \neq p$, since $p$ has performed its last write before $C_{\text {index }\left(\text { last }_{p}\right)}$. Suppose $w$ writes value $\left(\widehat{S_{x}}, x, n_{x}\right)$ into register $R$, and let $s$ be $x$ 's scan operation that precedes $w$ if it exists.

Suppose $\left(p, n_{p}\right) \in \widehat{S_{x}}$. Let $\mathcal{F}_{p}\left(C_{\ell}\right)=\mathcal{F}_{p}\left(C_{\ell-1}\right) \backslash\{R\}$ and $O_{p}\left(C_{\ell}\right)=O_{p}\left(C_{\ell-1}\right) \backslash\left\{v_{C_{\ell-1}}(R)\right.$.writer $\}$. If $R \in \mathcal{F}_{p}\left(C_{\ell-1}\right)$, then by definition, $v_{C_{\ell-1}}(R)$.writer $\in O_{p}\left(C_{\ell-1}\right)$ and if $R \notin \mathcal{F}_{p}\left(C_{\ell-1}\right)$, then by definition, $v_{C_{\ell-1}}(R)$.writer $\notin O_{p}\left(C_{\ell-1}\right)$. Since $\left|\mathcal{F}_{p}\left(C_{\ell-1}\right)\right|=\left|O_{p}\left(C_{\ell-1}\right)\right|,\left|\mathcal{F}_{p}\left(C_{\ell}\right)\right|=\left|O_{p}\left(C_{\ell}\right)\right|$. Therefore $(i)$ is true. Since $O_{p}\left(C_{\ell}\right) \subseteq O_{p}\left(C_{\ell-1}\right)$, (ii) holds. Since $\left(p, n_{p}\right) \in \widehat{S}_{x},(i i i)$ is true.

Now consider the case $\left(p, n_{p}\right) \notin \widehat{S_{x}}$. We first show that $C_{\text {index }(s)}$ precedes $C_{\text {index }\left(\text { last }_{p}\right)}$ in $\Gamma_{E}$ or $w$ is the first write by $x$. Suppose, for the purpose of contradiction, that $\operatorname{index}\left(\right.$ last $\left._{p}\right) \leq \operatorname{index}(s) \leq \ell-1$. First consider the case that there exists an $i$ such that $v_{C_{\text {index }(s)}}(R[i])$.writer $=p$. Since at $C_{\text {index }\left(\text { last }_{p}\right)}$ all registers contain $\left(\widehat{S_{p}}, p, n_{p}\right)$ and $p$ does not write after last $_{p}, v_{C_{\text {index }(s)}}(R[i])$.proposal $=n_{p}$. Hence by Line 21 $\left(p, n_{p}\right) \in \widehat{S_{x}}$. Otherwise suppose that in $C_{\text {index }(s)}$, there are at least two distinct registers whose writer are the same process and not $p$. Then, choose any indices $i, j$ such that $i<j$ and $v_{C_{\text {index }(s)}}(R[i])$.writer $=v_{C_{\text {index }(s)}}(R[j])$.writer $=u \neq p$. Let $w_{1}$ and $w_{2}$ be the most recent writes to $R[i]$ and $R[j]$ preceding $C_{\text {index }(s)}$. Hence $w_{1}$ has value $v_{C_{\text {index }(s)}}(R[i])$ and $w_{2}$ has value $v_{C_{\text {index }(s)}}(R[j])$ and they both are performed by process $u$. Furthermore, since at $C_{\operatorname{index}\left(\text { last }_{p}\right)}$ all registers contain $\left(\widehat{S_{p}}, p, n_{p}\right), w_{1}$ and $w_{2}$ occur in Interval $\left[\operatorname{index}\left(\right.\right.$ last $\left._{p}\right)$, index $\left.(s)\right]$. Suppose $v_{C_{\text {index }(s)}}(R[i]) \neq \mathrm{v}_{C_{\text {index }(s)}}(R[j])$, then by Lemma 6.1, $w_{1}$ precedes $w_{2}$ in $E$ and by the induction hypothesis $(i i i),\left(p, n_{p}\right) \in \mathrm{v}_{C_{\text {index }(s)}}(R[j])$.set. Otherwise suppose $v_{C_{\text {index }(s)}}(R[i])=v_{C_{\text {index }(s)}}(R[j])$ then again by induction hypothesis $(i i i),\left(p, n_{p}\right) \in \mathrm{v}_{C_{\text {index }(s)}}(R[j])$.set. In either case, when $x$ performs Line 24 after $s$ and preceding $w$, this line evaluates to true. Hence by Line 25, $\left(p, n_{p}\right) \in \widehat{S_{x}}$. Finally, if $\forall i, j, 1 \leq i, j \leq b$ and $i \neq$ $j, v_{C_{\text {index }(s)}}(R[i])$.writer $\neq v_{C_{\text {index }(s)}}(R[j])$.writer, then by induction hypothesis $(i),\left|\mathcal{F}_{p}\left(C_{\operatorname{index}(s)}\right)\right|=\left|O_{p}\left(C_{\operatorname{index}(s)}\right)\right|=b$. Therefore, by the for-loop (Lines 18, 22), $\left|\widehat{S_{x}}\right|+1 \geq\left|O_{p}\left(C_{\text {index }(s)}\right)\right|=b$. Hence, by Line ??, the presumed write $w$ by $x$ cannot happen. Thus, in all cases, we have established that if $\left(p, n_{p}\right) \notin \widehat{S_{x}}$ then $C_{\operatorname{index}(s)}$ precedes $C_{\operatorname{index}\left(\text { last }_{p}\right)}$ in $\Gamma_{E}$ or $w$ is the first write by $x$.

Consequently, any write by $x$ before $w$ happens before $C_{\text {index }\left(\text { last }_{p}\right)}$. On the other hand, by the induction hypothesis, for all $q \in O_{p}\left(C_{\ell-1}\right), q$ performs a write during Interval[index $\left(\right.$ last $\left.\left._{p}\right), \ell-1\right]$ implying $x$ is not in $O_{p}\left(C_{\ell-1}\right)$. Thus by defining $\mathcal{F}_{p}\left(C_{\ell}\right)=\mathcal{F}_{p}\left(C_{\ell-1}\right) \cup\{R\}$ and $O_{p}\left(C_{\ell}\right)=O_{p}\left(C_{\ell-1}\right) \backslash\left\{v_{C_{\ell-1}}(R)\right.$.writer $\} \cup\{x\}$, the induction hypothesis $(i)$ and (ii) hold for $\ell$. Since the most recent operation before $w$ by $x$ happens before $C_{\operatorname{index}\left(\text { last }_{p}\right)}, x$ performs only one write operation in Interval[index $\left(\right.$ last $\left.\left._{p}\right), \ell\right]$. Therefore, (iii) holds for $\ell$.

Lemma 6.3. Let $p$ and $q$ be two distinct processes that have terminated in execution $E$ and returned names $n_{p}$ and $n_{q}$ respectively. Suppose that $C_{\mathrm{index}\left(\text { last }_{p}\right)}$ precedes $C_{\mathrm{index}\left(\text { last }_{q}\right)}$ in $\Gamma_{E}$. If $n_{p}, n_{q} \leq b-1$, then $\left|\mathcal{F}_{p}\left(C_{\mathrm{index}\left(\text { last }_{q}\right)}\right)\right|=0$.

Proof. Since $n_{q} \leq b-1, q$ returns in Line 13. Hence $C_{\text {index }\left(l a s t_{q}\right)}$ is consistent with content $\left(S_{q}, q, n_{q}\right)$. Therefore, $\left.\mid \mathcal{F}_{p}\left(C_{\text {index }(\text { last }}\right)\right) \mid \in\{0, b\}$. By Lemma 6.2, $\left|\mathcal{F}_{p}\left(C_{\text {index }\left(\text { last }_{q}\right)}\right)\right|=\left|O_{p}\left(C_{\operatorname{index}\left(\text { last }_{q}\right)}\right)\right|$. Since in $\left.C_{\text {index }(\text { last }}\right)$, R.writer $=q$ for all $R \in \mathcal{R},\left|O_{p}\left(C_{\text {index }\left(\text { last }_{q}\right)}\right)\right| \leq 1$, and thus $\left|\mathcal{F}_{p}\left(C_{\text {index }\left(\text { last }_{q}\right)}\right)\right| \leq 1$. Therefore $\left|\mathcal{F}_{p}\left(C_{\text {index }\left(\text { last }_{q}\right)}\right)\right|=0$.

\section{Lemma 6.4. The names returned by any two distinct processes are distinct.}

Proof. For any two distinct processes $p$ and $q$, let $n_{p}$ and $n_{q}$ be the names returned by $p$ and $q$, respectively. Let $\widehat{S_{p}}$ (respectively, $\widehat{S_{q}}$ ) be the value of $S_{p}$ (respectively, $S_{q}$ ) when $p$ (respectively, $q$ ) executes Line 12. If $\left|\widehat{S_{p}}\right|,\left|\widehat{S_{q}}\right| \geq b-1$, the names returned by $p$ and $q$ in Line 15 are distinct because $p \neq q$.

Consider the case $\left|\widehat{S_{p}}\right| \leq b-2$ and $\left|\widehat{S_{q}}\right| \geq b-1$. Process $p$ returns $n_{p}$ in Line 13 . Since $\mid$ Names $\left(S_{p}\right)|\leq| S_{p} \mid$, by Line 5, $n_{p}$ must be smaller than or equal to $b-1$. Furthermore the name returned by $q$ in Line 15 is larger than or equal $b$. The case $\left|\widehat{S_{q}}\right| \leq b-2$ and $\left|\widehat{S_{p}}\right| \geq b-1$ is true by symmetry. Consider the case $\left|\widehat{S_{p}}\right|,\left|\widehat{S_{q}}\right| \leq b-2$ implying $n_{p}, n_{q} \leq b-1$. Without loss of generality assume that $C_{\operatorname{index}\left(\text { last }_{p}\right)}$ precedes $C_{\operatorname{index}\left(\text { last }_{q}\right)}$ in $\Gamma_{E}$. By Lemma 6.3 . 
$\left.\mid \mathcal{F}_{p}\left(C_{\text {index }(\text { last }}\right)\right) \mid=0$. Since $\forall R \in \mathcal{R}, v_{C_{\text {index }(\text { last } q)}}(R)$.writer $=q \neq p,\left(p, n_{p}\right) \in v_{C_{\text {index }(\text { last } q)}}(R)$.set. Thus by Line 23, $\left(p, n_{p}\right) \in \widehat{S_{q}}$. Therefore by Line 5 , proposed $_{q} \neq n_{p}$.

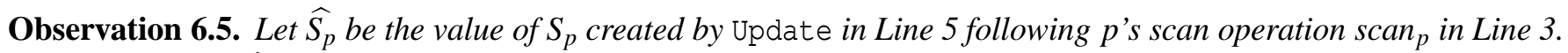
Then $\forall q \in \operatorname{Procs}\left(\widehat{S_{p}}\right)$, q performs at least one write before scan $_{p}$.

Lemma 6.6. Let $k$ be the number of participating processes during process p's get Name (). Then, the name returned by $p$, is in the range $\{1, \ldots, k\}$, if $k \leq b-1$ and in the range $\{1, \ldots, n+b-1\}$, if $k \geq b$.

Proof. Let $\widehat{S_{p}}$ be the value of $S_{p}$ when $p$ executes Line 4 for the last time and $n_{p}$ be the name returned by $p$. By Observation 6.5, $\forall q \in \operatorname{Procs}\left(\widehat{S_{p}}\right), q$ performs at least one write before $p$ returns. Thus, $\forall q \in \operatorname{Procs}\left(\widehat{S_{p}}\right), q$ is a participating process. Hence, $\left|\widehat{S_{p}}\right|+1 \leq k$.

If $k \leq b-1$, process $p$ returns in Line 13, By definition, $\left|\operatorname{Names}\left(\widehat{S_{p}}\right)\right| \leq\left|\widehat{S_{p}}\right| \leq k-1$. Therefore by Line 5 , $n_{p} \leq\left|\operatorname{Names}\left(\widehat{S_{p}}\right)\right|+1 \leq k$.

If $k \geq b$, then $p$ returns either in Line 13 or in Line 15 . Therefore the name is in the range $\{1, \ldots, b+n-1\}$.

Theorem 6.7. For any $b \geq 2$ there is an obstruction-free $(b-1)$-bounded $k$-adaptive renaming algorithm implemented from $b+1$ bounded registers such that when $k \geq b$ the returned names are in the range $\{1, \ldots, n+b-1\}$.

Proof. There is an obstruction-free implementation of $b$-component snapshot objects from $b+1$ bounded registers [22]. Since our algorithm in Fig. 3 is deterministic we can replace the atomic scan registers with a linearizable scan. By Lemma 6.6 and Lemma 6.4, the algorithm solves $(b-1)$-bounded $k$-adaptive renaming. Thus, it suffices to prove that the algorithm is obstruction-free.

If $p$ runs alone then the value of $S_{p}$ computed in Line 4 and proposed ${ }_{p}$ computed in Line 5 remain the same. Therefore after $b$ write operations all registers contain $\left(S_{p}, p\right.$, proposed $\left.{ }_{p}\right)$. Therefore, in the $b$-th iteration of the repeat-until loop (Line 11) evaluates to true and $p$ stops.

Let $f:\{1, \ldots, n\} \rightarrow \mathbb{N}$ be a non-decreasing function where, $\forall k \in\{1, \ldots, n\}, f(k) \geq k$ and $f(1) \leq n-1$. Let $d^{\prime}=\min \{n, x \mid f(x) \geq 2 n\}$. Hence, $d^{\prime} \leq n$. Setting $b=d^{\prime}$, we have an obstruction-free one-shot $d^{\prime}$-bounded $k$-adaptive renaming algorithm from $d^{\prime}+1$ registers. This implies that the algorithm returns names in the range $\{1, \ldots, k\}$ when $k \leq d^{\prime}-1$, and returns names in the range $\left\{1, \ldots, n+d^{\prime}-1\right\}$ when $k \geq d^{\prime}$. Note that $\forall k \in\left\{1, \ldots, d^{\prime}-1\right\}, k \leq f(k)$. Furthermore, when $k \geq d^{\prime}, n+d^{\prime}-1<2 n \leq f\left(d^{\prime}\right)$. Hence, $\forall k \in\{1, \ldots, n\}$, the algorithm returns names in the range $\{1, \ldots, f(k)\}$.

Corollary 6.1. There is an obstruction-free one-shot $f$-adaptive renaming algorithm implemented from $\min \{n, x \mid f(x) \geq 2 n\}+1$ bounded registers.

\section{Observations and Open Problems}

Let $f:\{1, \ldots, n\} \rightarrow \mathbb{N}$ be a non-decreasing function where, $\forall k \in\{1, \ldots, n\}, f(k) \geq k$ and $f(1) \leq n-1$. Let $=\max \{x \mid f(x) \leq n-1\}$. We proved a lower bound of +1 for non-deterministic solo-terminating long-lived $f$ adaptive renaming. Furthermore, for any integer constant $0 \leq c \leq n$, we showed a lower bound of $\left\lfloor\frac{2(n-c)}{c+2}\right\rfloor$ for non-deterministic solo-terminating one-shot $(k+c)$-adaptive renaming. This implies a tight space bound of $n$ for both one-shot and long-lived tight renaming. We also presented an obstruction-free one-shot $f$-adaptive algorithm from $\min \{n, x \mid f(x) \geq 2 n\}+1$ registers.

An obvious solution for any obstruction-free long-lived or one-shot $f$-adaptive renaming is as follows. A set $Q \subseteq \mathcal{P}$ of $\lfloor f(1)\rfloor-1$ processes always return names in the range $\{1, \ldots, \max (\lfloor f(1)\rfloor-1,1)\}$ without taking any steps. In any $(\mathcal{P} \backslash Q)$-solo execution, process in $\mathcal{P} \backslash Q$ using universal construction, get names in the range $\{\lfloor f(1)\rfloor, \ldots, k+$ $\lfloor f(1)\rfloor-1\}$. Universal construction for $|\mathcal{P} \backslash Q|$ processes requires $|\mathcal{P}|-|Q|=n-\lfloor f(1)\rfloor+1$ registers. Observe that 
this is a tight upper bound for obstruction-free long-lived $(k+c)$-adaptive renaming. One of the most noticeable open problems is whether implementing one-shot $f$-adaptive renaming requires asymptotically less space than long-lived $f$-adaptive renaming.

We designed a wait-free one-shot $(b-1)$-bounded $(k(k+1) / 2)$-adaptive renaming algorithm from $b$ bounded registers, and established that this algorithm has a polynomial step complexity. It appears that if we modify the newScan () function of our algorithm, so that each process returns when the set of all processes know to it grows even by one, the step complexity would reduce considerably. However this change would require much more elaborate and challenging proofs because the values returned by newScan () would not be equivalent to values returned by a linearizable scan.

For some systems, it seems reasonable to have the register space, as well as the name space, adapt to the actual number of participants. The one-shot lower bound can also be modified to express the actual register use as a function of $k$. On the other hand, the one-shot algorithms in this paper require a fixed number of registers regardless of the number of participants.

\section{References}

[1] Y. Afek and M. Merritt. Fast, wait-free (2k-1)-renaming. In Proc. of 18th PODC, pages 105-112. Journal of the ACM, 1999.

[2] D. Alistarh, J. Aspnes, K. Censor-Hillel, S. Gilbert, and M. Zadimoghaddam. Optimal-time adaptive strong renaming, with applications to counting. In Proc. of 30th PODC, pages 239-248, 2011.

[3] D. Alistarh, J. Aspnes, S. Gilbert, and R. Guerraoui. The complexity of renaming. In Proc. of 52nd FOCS, pages 718-727, 2011.

[4] D. Alistarh, H. Attiya, S. Gilbert, A. Giurgiu, and R. Guerraoui. Fast randomized test-and-set and renaming. In Proc. of 24th DISC, pages 94-108, 2010.

[5] H. Attiya, A. Bar-Noy, D. Dolev, D. Peleg, and R. Reischuk. Renaming in an asynchronous environment. Journal of the ACM, 37(3):524-548, 1990.

[6] H. Attiya, F. Fich, and Y. Kaplan. Lower bounds for adaptive collect and related objects. In Proc. of 23rd PODC, pages 60-69, 2004.

[7] H. Attiya and A. Paz. Counting-based impossibility proofs for renaming and set agreement. In Proc. of 26th DISC, pages 356-370, 2012.

[8] H. Attiya and J. Welch. Distributed Computing: Fundamentals, Simulations and Advanced Topics. John Wiley Interscience, 2004.

[9] E. Borowsky and E. Gafni. Generalized flp impossibility result for t-resilient asynchronous computations. In Proc. of 25th ACM STOC, pages 91-100, 1993.

[10] A. Brodsky, F. Ellen, and P. Woelfel. Fully-adaptive algorithms for long-lived renaming. Journal of Distributed Computing, 24(2):119-134, 2011.

[11] J. Burns and N. Lynch. Bounds on shared memory for mutual exclusion. Journal of Information and Computation, 107(2):171-184, 1993.

[12] J. Burns and G. Peterson. The ambiguity of choosing. In Proc. of 8th PODC, PODC '89, pages 145-157. Journal of the ACM, 1989.

[13] A. Castañeda and S. Rajsbaum. New combinatorial topology upper and lower bounds for renaming. In Proc. of 27th PODC, pages 295-304, 2008. 
[14] Danny Dolev and Nir Shavit. Bounded concurrent time-stamping. SIAM Journal on Computing, 26(2):418-455, 1997.

[15] Cynthia Dwork and Orli Waarts. Simple and efficient bounded concurrent timestamping and the traceable use abstraction. Journal of the ACM, 46(5):633-666, 1999.

[16] W. Eberly, L. Higham, and J. Warpechowska-Gruca. Counting-based impossibility proofs for renaming and set agreement. In Proc. of 26th DISC, pages 149-160, 1998.

[17] F. Ellen and P. Woelfel. An optimal implementation of fetch-and-increment. In Proc. of 27th DISC, pages 284-298, 2013.

[18] F. Fich, M. Herlihy, and N. Shavit. On the space complexity of randomized synchronization. Journal of the ACM, pages 843-862, 1998.

[19] F. Fich and E. Ruppert. Hundreds of impossibility results for distributed computing. Distributed Computing, 16(2-3):121-163, 2003.

[20] E. Gafni. Read-write reductions. In Proceedings of the 8th International Conference on Distributed Computing and Networking, pages 349-354, 2006.

[21] Rainer Gawlick, Nancy A. Lynch, and Nir Shavit. Concurrent timestamping made simple. In 1st Israel Symposium on Theory of Computing Systems (ISTCS), pages 171-183, 1992.

[22] G. Giakkoupis, M. Helmi, L. Higham, and P. Woelfel. An O(sqrt n) space bound for obstruction-free leader election. In Proc. of 31st DISC, pages 46-60, 2013.

[23] M. Helmi, L. Higham, E. Pacheco, and P. Woelfel. The space complexity of long-lived and one-shot timestamp implementations. Journal of the ACM, 61(1):7-27, 2014.

[24] M. Herlihy and N. Shavit. The topological structure of asynchronous computability. Journal of the ACM, 46(6):858-923, 1999.

[25] Amos Israeli and Ming Li. Bounded time-stamps. Distributed Computing, 6(4):205-209, 1993.

[26] Amos Israeli and Meir Pinhasov. A concurrent time-stamp scheme which is linear in time and space. In Distributed Algorithms, 6th International Workshop (WDAG), pages 95-109, 1992.

[27] N. Lynch. Distributed Algorithms. Morgan Kaufmann, 1996.

[28] M. Moir and J. Garay. Fast, long-lived renaming improved and simplified. In Procedeeding of the 10th International Workshop on Distributed Algorithms (WDAG), pages 287-303, 1996.

[29] A. Panconesi, M. Papatriantafilou, P. Tsigas, and P. Vitnyi. Randomized naming using wait-free shared variables. Journal of Distributed Computing, 11:113-124, 1998. 